\title{
TYPE II SINGULARITIES ON COMPLETE NON-COMPACT YAMABE FLOW
}

\author{
BEOMJUN CHOI, PANAGIOTA DASKALOPOULOS, AND JOHN KING
}

\begin{abstract}
This work concerns with the existence and detailed asymptotic analysis of Type II singularities for solutions to complete non-compact conformally flat Yamabe flow with cylindrical behavior at infinity. We provide the specific blow-up rate of the maximum curvature and show that the solution converges, after blowing-up around the curvature maximum points, to a rotationally symmetric steady soliton. It is the first time that the steady soliton is shown to be a finite time singularity model of the Yamabe flow.
\end{abstract}

\section{Contents}

1. Introduction and preliminaries 1

2. Formal matched asymptotics 5

3. Notation and different scalings 8

\begin{tabular}{ll|l}
3.1. & Coordinate systems & 8
\end{tabular}

$\begin{array}{llr}3.2 . & \text { Scalings } & 8\end{array}$

$\begin{array}{lll}3.3 . & \text { Relations } & 8\end{array}$

3.4. Functions 9

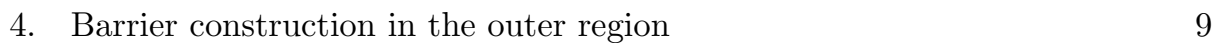

$\begin{array}{lll}5 . & \text { Barrier construction in the inner region } & 18\end{array}$

6. Construction of super and sub-solutions 19

7. Asymptotic shape of the singularity in the inner region and geometric properties 24

8. Proof of Theorem 1.2 30

References 35

\section{INTRODUCTION AND PRELIMINARIES}

Let $\left(M, g_{0}\right)$ be a Riemannian manifold without boundary of dimension $n \geq 3$. For a mertric

$$
g=u^{\frac{4}{n+2}} g_{0}
$$

which is conformal to $g_{0}$, the scalar curvature $R$ of $g$ is given in terms of the scalar curvature $R_{0}$ of $g_{0}$ by

$$
R=u^{-1}\left(-\bar{c}_{n} \Delta_{g_{0}} u^{\frac{n-2}{n+2}}+R_{0} u^{\frac{n-2}{n+2}}\right)
$$

where $\Delta_{g_{0}}$ denotes the Laplace Beltrami operator with respect to $g_{0}$ and $\bar{c}_{n}=$ $4(n-1) /(n-2)$. 
In 1989 R. Hamilton introduced the Yamabe flow

$$
\frac{\partial g}{\partial t}=-R g
$$

as an approach to solve the Yamabe problem on manifolds of positive conformal Yamabe invariant. In the case where $M$ is compact the long time existence and convergence of Yamabe flow is well understood. Hamilton $[\mathrm{H}]$ himself showed the existence of the normalized Yamabe flow (which is the re-parametrization of (1.1) to keep the volume fixed) for all time; moreover, in the case when the scalar curvature of the initial metric is negative, he showed the exponential convergence of the flow to a metric of constant scalar curvature. Chow $\mathrm{Ch}$ showed the convergence of the flow, under the conditions that the initial metric is locally conformally flat and of positive Ricci curvature. The convergence of the flow for any locally conformally flat initially metric was shown by $\mathrm{Ye}$ Ye.

Schwetlick and Struwe [SS] obtained the convergence of the Yamabe flow on a general compact manifold under a suitable Kazdan-Warner type of condition that rules out the formation of bubbles and that is verified (via the positive mass Theorem) in dimensions $3 \leq n \leq 5$. The convergence result, in its full generality, was established by Brendle [B1] and [B2] (up to a technical assumption, in dimensions $n \geq 6$, on the rate of vanishing of Weyl tensor at the points at which it vanishes): starting with any smooth metric on a compact manifold, the normalized Yamabe flow converges to a metric of constant scalar curvature.

Although the Yamabe flow on compact manifolds is well understood, the complete non-compact case is unsettled. In this case one expects to have more types of singularities which could be either of type I or type II according to the definition below.

Definition 1.1. Assume that a solution $g(t)$ of the Yamabe flow (1.1) on a Riemannian manifold has a singularity at time $T$. This singularity is called type $I$ if

$$
\limsup _{t \rightarrow T^{-}}(T-t) \sup _{M}|R m|(\cdot, t)<+\infty .
$$

A singularity which is not of type $I$, is called type II.

The results mentioned above show that in the generic compact case the only singularities of the Yamabe flow are type I. Moreover the works [DS1, DKS] address the singularity formation of complete non-compact solutions to the conformally flat Yamabe flow whose conformal factors have cylindrical behavior at infinity. These singularities are all of type I.

A natural question to ask is whether the Yamabe flow admits any singularities which are of type II in the non-compact case. The authors in CD presented, for the first time in the Yamabe flow, examples of complete solutions which develop a type II singularity, either at finite time $T<+\infty$ or at infinite time $T=+\infty$. Such solutions are conformally equivalent to $\mathbb{R}^{n}$ and their initial data has cylindrical behavior at infinity if $T<\infty$. What distinguishes our type II solutions from the type I solutions which are modeled on shrinkers, is that their initial metric has slower second order decay rate to the cylindrical metric than that of any other Yamabe shrinkers. In this work we study complete non-compact and conformally flat solutions of the Yamabe flow (1.1) on $\mathbb{R}^{n}$ which develop type II singularity and provide their detailed asymptotic behavior near the singularity. 
Let us briefly discuss next the known results on the singularity formation of non-compact Yamabe flow. Even though the analogue of Perelman's monotonicity formula is still lacking for the Yamabe flow, one expects that Yamabe soliton solutions model finite and infinite time singularities. These are special solutions of the Yamabe flow (1.1) which is characterized by a metric $g=g_{i j}$ and a potential function $P$ so that

$$
(R-\rho) g_{i j}=\nabla_{i} \nabla_{j} P, \quad \rho \in\{1,-1,0\} .
$$

Depending on the sign of the constant $\rho$, a Yamabe soliton is called a Yamabe shrinker, a Yamabe expander or a Yamabe steady soliton if $\rho=1,-1$ or 0 respectively.

The classification of locally conformally flat Yamabe solitons with positive sectional curvature was established in [DS2] (c.f. also [CSZ] and [CMM]). It is shown in DS2 that such solitons are globally conformally equivalent to $\mathbb{R}^{n}$ and correspond to radially symmetric self-similar solutions of the fast-diffusion equation

$$
u_{t}=\frac{n-1}{m} \Delta u^{\frac{n-2}{n+2}}, \quad \text { on } \mathbb{R}^{n} \times[0, T)
$$

satisfied by the conformal factor defined by $g_{i j}=u^{\frac{4}{n+2}} \delta_{i j}$. Here and in the sequel $\delta_{i j}$ denotes the standard metric on $\mathbb{R}^{n}$ and we set $m:=(n-2) /(n+2)$. A complete description of those solutions is given in DS2. In CSZ the assumption of positive sectional curvature was relaxed to that of nonnegative Ricci curvature.

As mentioned above, in DS1, DKS] the singularity formation of complete noncompact solutions to the conformally flat Yamabe flow with cylindrical behavior at infinity was studied. The singularity profiles are Yamabe shrinking solitons which are determined by the second order asymptotics at infinity of the initial data, which is matched with that of the corresponding soliton. The solutions may become extinct at the extinction time $T$ of the cylindrical tail or may live longer than $T$. In the first case, the singularity profile is described by a Yamabe shrinker that becomes extinct at time $T$. This result can be seen as a stability result around the Yamabe shrinkers with cylindrical behavior at infinity. In the second case, the flow develops a singularity at time $T$ which is described by a singular Yamabe shrinker slightly before $T$ and by a matching Yamabe expander slightly after $T$.

Recently the authors [CD studied long time behavior of the complete noncompact conformally flat Yamabe flow and in particular showed the stability around the steady solitons. Such solitons are conformally equivalent to $\mathbb{R}^{n}$ and rotationally symmetric. They are the analogue to the bowl translating soliton of MCF or the Bryant soliton of the Ricci flow.

In this work we study the asymptotic behavior of type II singularities in the conformally flat non-compact case. More precisely, for a sufficiently small $T<$ $+\infty$, we provide a condition, in terms of the second order decay rate of the initial metric $g_{\gamma, \kappa}(\cdot, 0)$ at spatial infinity, which guarantees that the Yamabe flow $g_{\gamma, \kappa}(\cdot, t)$ develops a type II singularity at time $T$ with specified blow up rate

$$
\limsup _{t \rightarrow T^{-}}(T-t)^{1+\gamma} \sup _{M}|R m|(\cdot, t)=\kappa .
$$

Moreover, we prove that after rescaling the solution $g_{\gamma, \kappa}(\cdot, t)$ around highest curvature point by $(T-t)^{-(1+\gamma)}$, it converges to a radial steady gradient soliton.

Our main result states as follows: 
Theorem 1.2. Let $g_{0}=u_{0}^{1-m}(x) \delta_{i j}$ be a conformally flat metric with positive Ricci curvature. For any given $\gamma>0$ and $A>0$, there is $T_{1}>0$ with the following property: for any $T<T_{1}$, if

i) $u_{0}^{1-m}(x)<\frac{(n-1)(n-2)}{|x|^{2}} T, \forall x \in \mathbb{R}^{n}$, and

ii) $u_{0}^{1-m}(x)=\frac{(n-1)(n-2)}{|x|^{2}}\left(T-\left(\frac{\ln |x|}{A}\right)^{-\frac{1}{\gamma}}+O\left((\ln |x|)^{-\frac{1}{\gamma}-1}\right)\right)$, as $|x| \rightarrow+\infty$

then the solution of Yamabe flow (1.1) with initial data $g_{0}$ will develop a type II singularity at time $t=T$ with specified blow up rate given by

$$
\limsup _{t \rightarrow T^{-}}(T-t)^{1+\gamma} \sup _{M}|\operatorname{Rm}|(\cdot, t)=\frac{2 \gamma A}{\sqrt{n(n-1)}} .
$$

Moreover, after rescaling the metric around the highest curvature point by ( $T-$ $t)^{-(1+\gamma)}$, it converges to the unique radial steady gradient soliton of maximum scalar curvature $2 \gamma A$.

Theorem 1.2 shows for the first time that the conformally flat radial steady soliton appears as a finite time singularity model for the Yamabe flow. In the Mean curvature flow and the Ricci flow, examples of type II singularities and their asymptotic behavior were shown in both compact and non-compact settings under rotational symmetry (c.f. AV], IW] for Mean curvature flow and AIK2, AIK1, [W] for Ricci flow). Let us remark that unlike in the cases mentioned above our result also includes non-radial initial data.

To achieve our result we first construct sharp barriers (super and sub-solutions) for given fixed $\gamma$ and $A$. The barriers are chosen sufficiently close to each other so that they give a model solution whose blow up limit at the tip is a radial steady soliton with the curvature blow up rate (1.4). In other words this already proves the result of Theorem 1.2 if the initial metric is in between sharp barriers. When the initial metric satisfies the condition of Theorem 1.2 , the solution can be located between two model solutions which are differ by a translation in cylindrical direction. Then we do further analysis to show the solution also has the same asymptotic behavior and curvature blow up rate.

The barriers will be constructed to be radially symmetric though we don't assume it for initial metrics. Note that for a radially symmetric solution $g:=$ $u^{\frac{4}{n+2}}(r, t)\left(d r^{2}+r^{2} d g_{S^{n-1}}\right)$ of the Yamabe flow 1.2$)$, it is often convenient to work in cylindrical coordinates where the metric is expressed as $g=w(s, t)\left(d s^{2}+d g_{s^{n-1}}\right)$, with $s=\ln r$. The conformal factor in cylindrical coordinates is given by

$$
w(s, t)=r^{2} u^{\frac{4}{n+2}}(r, t), \quad s=\ln r
$$

and satisfies the equation

$$
\frac{m}{n-1}\left(w^{\frac{n+2}{4}}\right)_{t}=\left(w^{\frac{n-2}{4}}\right)_{s s}-\left(\frac{n-2}{2}\right)^{2} w^{\frac{n-2}{4}}, \quad \text { on } \mathbb{R} \times[0, T]
$$

with $m:=\frac{n-2}{n+2}$.

The outline of our paper is as follows: In the Section 2 we will begin by giving the formal matched asymptotics of the type II singularity at time $t=T$. Based on this analysis, we will introduce in Section 3 the two different regions outer and inner and the scalings in each region. Also some notation. We will refer to the notation of this section throughout the paper. Section 4 deals with the barrier 
construction in the outer region (c.f. Propositons 4.4 and 4.7) and Section 5 deals with the barrier construction in the inner region (c.f. Proposition 5.1). Combining the results from Sections 4 and 5 , in Section 6 we will glue the barriers in the inner and outer region to construct suitable super and sub-solutions. In Section 7 we will show one of our main results, Theorem 7.1, which shows the type II convergence of any given conformally flat Yamabe flow to the steady soliton, assuming that its initial data is trapped between our super and sub-solutions. Finally, our last section 8 will be devoted to the proof of Theorem 1.2. In this section, along with the barriers constructed in previous sections, we will make use of the differential Harnack inequality in $\mathrm{Ch}$, and the classification result of Yamabe solitons (c.f. [DS2, CMM, CSZ]).

\section{Formal matched asymptotics}

In this section we will present the formal construction of our solutions which is based on matched asymptotic analysis. We hope that this will give our reader an intuition for our construction.

For any given parameters $\gamma>0$ and $A>0$ we will construct below a family of formal rotationally symmetric solutions where the curvature blows up in the type II rate

$$
\lim _{t \rightarrow T^{-}}(T-t)^{1+\gamma} \sup _{M_{t}}|\operatorname{Rm}|=\frac{2 \gamma A}{\sqrt{n(n-1)}} .
$$

Note that our main results Theorem 6.4 and 7.1 are not restricted on rotationally symmetric solutions, however the barriers which will be constructed in next sections are obtained from perturbations of this formal solution and those are radial.

Motivated by condition 2.1] and in order to capture at the end a stationary solution, we perform the change of variables on our solution $w(s, t)$ of 1.6 setting

$$
\hat{w}(\eta, \tau)=(T-t)^{-1} w(s, t), \quad \eta=(T-t)^{\gamma} s, \tau=-\ln (T-t) .
$$

A direct calculation shows that $\hat{w}(\eta, \tau)$ satisfies the evolution

$$
B[\hat{w}]=0
$$

where

$$
\begin{aligned}
B[\hat{w}]:=\partial_{\tau} \hat{w}-(n-1) & e^{-2 \gamma \tau}\left(\frac{\hat{w}_{\eta \eta}}{\hat{w}}+\frac{n-6}{4} \frac{\hat{w}_{\eta}^{2}}{\hat{w}^{2}}\right) \\
& -\left(\gamma \eta \hat{w}_{\eta}+\hat{w}-(n-1)(n-2)\right) .
\end{aligned}
$$

Thus, if we assume that

$$
\partial_{\tau} \hat{w} \quad \text { and } \quad e^{-2 \gamma \tau}\left(\frac{\hat{w}_{\eta \eta}}{\hat{w}}+\frac{n-6}{4} \frac{\hat{w}_{\eta}^{2}}{\hat{w}^{2}}\right)
$$

are negligible as $\tau \rightarrow \infty$, the above equation is reduced to the following ODE in $\eta$ variable

$$
\gamma \eta \hat{w}_{\eta}+\hat{w}-(n-1)(n-2)=0 .
$$

Solving this equation on $\eta>0$ gives the solution $\hat{w}_{0}(\eta)$ given by

$$
\hat{w}_{0}(\eta):=(n-1)(n-2)\left(1-\kappa \eta^{-1 / \gamma}\right)
$$

for a parameter $\kappa \in \mathbb{R}$. We will assume from now on, without loss of generality, that $\kappa>0$, although $\kappa \leq 0$ also gives a solution. Moreover, there is a family of solutions to (2.6) on $\eta<0$, but this case is exactly symmetric to the $\eta>0$ case which 
we will handle below. Indeed, the solutions given by (2.7) describe non-compact surfaces moving in positive $\eta$ (hence positive $s$ ) direction, while the corresponding solutions defined on $\eta<0$ describe a symmetric surface just moving on the opposite direction. This ansatz, namely setting

$$
\hat{w}(\eta, \tau):=(n-1)(n-2)\left(1-\kappa \eta^{-\frac{1}{\gamma}}\right) \quad \text { on } \eta>\kappa^{\gamma}
$$

approximates a solution of the equation 2.3). In fact, plugging $\hat{w}(\eta, \tau)$ given by (2.8) into (2.3), we see that the error term becomes

$$
B[\hat{w}]=-(n-1) e^{-2 \gamma \tau}\left(\frac{\hat{w}_{\eta \eta}}{\hat{w}}+\frac{n-6}{4} \frac{\hat{w}_{\eta}^{2}}{\hat{w}^{2}}\right) .
$$

Writing $B[\hat{w}]=(n-1)\left(\hat{w} e^{\gamma \tau}\right)^{-2}\left(\hat{w} \hat{w}_{\eta \eta}+\frac{n-6}{4} \hat{w}_{\eta}^{2}\right)$, we see that it becomes arbitrarily small in the space-time region

$$
\left(e^{\gamma \tau} \hat{w}\right)^{-1}=o(1), \quad \text { as } \tau \rightarrow+\infty
$$

which we call the outer region (see Figure 1 below). This is the region where the diffusion doesn't play an important role and the remaining advection and reaction terms are dominant.

The inner-region is given by

$$
e^{\gamma \tau} \hat{w}(\eta, \tau)=O(1), \quad \text { as } \tau \rightarrow+\infty
$$

(see Figure 1). In this region we perform another scaling, setting

$$
\bar{w}(\xi, \tau)=e^{\gamma \tau} \hat{w}(\eta, \tau), \quad \eta=A+e^{-\gamma \tau} \xi
$$

for some choice of $A>0$, which combined with 2.2 gives

$$
\bar{w}(\xi, \tau)=e^{\gamma \tau} \hat{w}\left(A+e^{-\gamma \tau} \xi, \tau\right)=e^{(1+\gamma) \tau} w\left(A e^{\gamma \tau}+\xi, T-e^{-\tau}\right) .
$$

The evolution equation for $\bar{w}(\xi, \tau)$ is given by

$$
\begin{aligned}
I[\bar{w}]:= & e^{-\gamma \tau}\left(\bar{w}_{\tau}-(1+\gamma) \bar{w}\right)-(n-1)\left(\frac{\bar{w}_{\xi \xi}}{\bar{w}}+\frac{n-6}{4} \frac{\bar{w}_{\xi}^{2}}{\bar{w}^{2}}\right) . \\
& +(n-1)(n-2)-\gamma A \bar{w}_{\xi}=0
\end{aligned}
$$

Thus assuming that in this region the first term having $e^{-\gamma \tau}$ becomes negligible as $\tau \rightarrow \infty$, the equation is reduced to

$$
(n-1)\left(\frac{\bar{w}_{\xi \xi}}{\bar{w}}+\frac{n-6}{4} \frac{\bar{w}_{\xi}^{2}}{\bar{w}^{2}}\right)-(n-1)(n-2)+\gamma A \bar{w}_{\xi}=0 .
$$

This can be seen as the equation satisfied by a traveling wave solution of (1.6) with speed $\gamma A$. For each $A>0$, this equation admits a $\tau$ independent entire solution $\bar{w}(\xi)$ which is unique up to translation in $\xi$. For such profile $\bar{w}$, the function $\bar{w}(s-\gamma A t)$ becomes a traveling wave solution of equation (1.6). From the geometric point of view, $\bar{w}(s)$ corresponds to a radially symmetric non-compact metric on $\mathbb{R}^{n}$ via 1.5 and solutions with different $A$ 's are the only radially symmetric steady gradient solitons on locally conformally flat manifolds (c.f. [CSZ, DS2]). For such a solution $\bar{w}$, the highest curvature point is at the origin (i.e. $s=-\infty$ ) and one may formally compute that $|\operatorname{Rm}|_{\max }(t)=\frac{2 \gamma A}{\sqrt{n(n-1)}}$, hence leading to 2.1. 


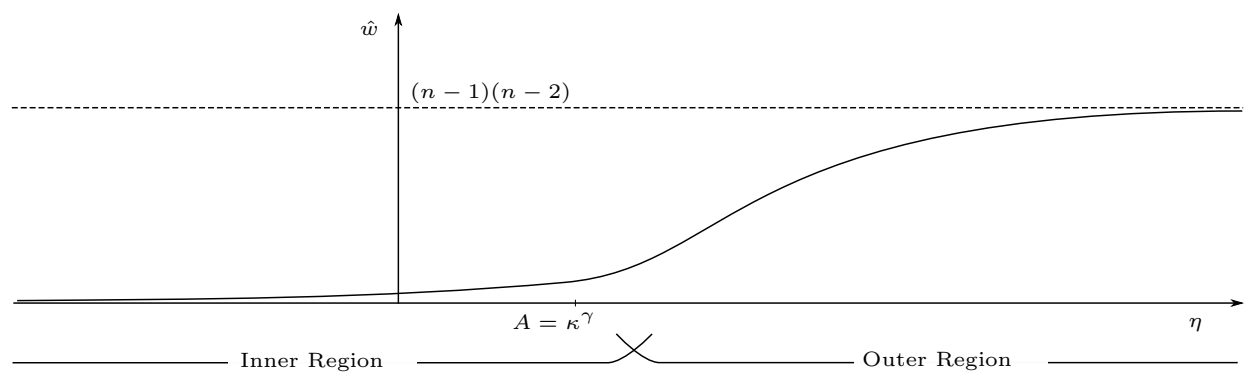

Figure 1

Remark 2.1. Note that a solution $\bar{w}(\xi, \tau)$ of 2.14 which also depends on $\tau$, can be written as

$$
\bar{w}(\xi, \tau)=\bar{w}_{0}(\xi+C(\tau))
$$

for a function $C(\tau)$, where $\bar{w}_{0}$ is one $\tau$-independent solution of (2.14). By plugging this into 2.13 again, we get an error term

$$
e^{-\gamma \tau}\left((1+\gamma) \bar{w}-C^{\prime}(\tau) \bar{w}_{\xi}\right) \approx 0 .
$$

We may choose $C(\tau)$ so that $C^{\prime}$ is small and thus the error term above vanishes appropriately as $\tau \rightarrow \infty$. Later, we will use this $C(\tau)$ to glue barriers from the two different regions, inner and outer.

Next, we will carry out a matching asymptotic analysis between the inner and outer regions and obtain a relation between $\kappa>0$ in (2.7) and $A>0$ in (2.13). It is known that a solution $\bar{W}(\xi)$ of 2.14 satisfies the asymptotic behavior

$$
\bar{W}(\xi) \approx \frac{(n-1)(n-2)}{\gamma A} \xi+O(1), \quad \text { as } \xi \rightarrow \infty .
$$

Therefore, recalling that $\eta=A+e^{-\gamma \tau} \xi$, our solution $\bar{w}(\xi, \tau)$ which is approximately $\bar{W}(\xi)$ satisfies

$$
e^{-\gamma \tau} \bar{w}(\xi, \tau) \approx \frac{(n-1)(n-2)}{\gamma A}(\eta-A)+o(1), \quad(\eta-A) e^{\gamma \tau} \gg 1, \tau \gg 1 .
$$

On the other hand, from the outer region ansatz (2.7), by the first order Taylor approximation near $\eta=\kappa^{\gamma}$ we have

$$
\begin{aligned}
\hat{w}(\eta, \tau) & \approx(n-1)(n-2) \frac{\kappa}{\gamma}\left(\kappa^{\gamma}\right)^{-\frac{1}{\gamma}-1}\left(\eta-\kappa^{\gamma}\right)+o(1) \\
& \approx \frac{(n-1)(n-2)}{\gamma \kappa^{\gamma}}\left(\eta-\kappa^{\gamma}\right)+o(1), \quad \text { for } \eta \text { near } \kappa^{\gamma}, \tau \gg 1 .
\end{aligned}
$$

Thus, we can see that these two asymptotics are matched exactly if

$$
A=\kappa^{\gamma} \text {. }
$$

To see this in another way, we can argue that if $A<\kappa^{\gamma}$ the two asymptotics are inconsistent as $\eta \rightarrow\left(\kappa^{\gamma}\right)_{+}$and if $\kappa^{\gamma}<A$ the linearization of (2.7) near $\eta=A$ is inconsistent with the asymptotic behavior from the inner region. 


\section{Notation AND DifFEREnT SCALINGS}

In this section we will summarize the coordinates and different scalings of our solutions, as introduced in the previous section. We will refer to the notation introduced below throughout the paper.

3.1. Coordinate systems. Since our metric $g(t)$ is conformally equivalent to the standard euclidean metric on $\mathbb{R}^{n}$ denoted briefly by $\delta_{i j}$, we represent

$$
g(t)=u^{1-m}(x, t) \delta_{i j}
$$

where the scalar function $u(x, t)$ evolves by equation 1.2 under Yamabe flow.

In case when the metric is radial, it is often convenient to work in the cylindrical coordinates, that is

$$
g(t)=u^{1-m}(r, t)\left(d r^{2}+r^{2} g_{S^{n-1}}\right)=w(s, t)\left(d s^{2}+g_{S^{n-1}}\right), \quad r=|x|
$$

and

$$
w(s, t)=r^{2} u^{1-m}(r, x), \quad s=\ln r=\ln |x| .
$$

Under this coordinate change, $w(s, t)$ evolves by equation 1.6 .

3.2. Scalings. We use the following different scaling in different regions:

- In the outer region, the conformal factor is represented by $\hat{w}(\eta, \tau)$ and is scaled from $w(s, t)$ as follows

$$
\hat{w}(\eta, \tau)=e^{\tau} w\left(e^{\gamma \tau} \eta, T-e^{-\tau}\right)
$$

The function $\hat{w}(\eta, \tau)$ evolves by the equation 2.3 .

- In the inner region, the conformal factor is represented by $\bar{w}(\xi, \tau)$ and is scaled from previous factors as follows

$$
\bar{w}(\xi, \tau)=e^{\gamma \tau} \hat{w}\left(A+\xi e^{-\gamma \tau}, \tau\right)=e^{(1+\gamma) \tau} w\left(A e^{\gamma \tau}+\xi, T-e^{-\tau}\right) .
$$

The function $\bar{w}(\xi, \tau)$ evolves by the equation 2.13).

- The above scaling change from $w(s, t)$ to $\bar{w}(\xi, \tau)$ corresponds to the following scaling change in euclidean coordinates from $u^{1-m}(x, t)$ to $\bar{u}^{1-m}(y, l)$ :

$$
|x|^{2} u^{1-m}(x, t)=(T-t)^{1+\gamma}|y|^{2} \bar{u}^{1-m}(y, l)
$$

where $l$ is a new time variable $l=(T-t)^{-\gamma} / \gamma$ and

$$
x=y e^{\gamma A l}=y e^{A(T-t)^{-\gamma}} .
$$

This scaling is used only in Section 7, $\bar{u}(y, l)$ evolves by equation 7.1).

3.3. Relations. Let us summarize relations between different variables and functions appearing in the scalings introduced above.

- We use three time scales:

$$
t \in[0, T), \quad \tau=-\ln (T-t), \quad l=\frac{(T-t)^{-\gamma}}{\gamma}=\frac{e^{\gamma \tau}}{\gamma} .
$$

The last one will only be used in the last section.

- The three space scales in cylindrical coordinates are:

$$
s \in \mathbb{R}, \quad \eta:=e^{-\gamma \tau} s, \quad \xi=s-A e^{\gamma \tau}=(\eta-A) e^{\gamma \tau} .
$$

- The corresponding three conformal factors in cylindrical coordinates at the different scales are:

$$
w(s, t)=e^{-\gamma \tau} \hat{w}(\eta, \tau)=e^{-(1+\gamma) \tau} \bar{w}(\xi, \tau) .
$$


In the radial case, $w(\xi, \tau)$ and $\bar{u}^{1-m}(y, l)$ given by 3.1 are related by

$$
\bar{w}(\xi, \tau)=|y|^{2} \bar{u}(y, l), \quad \xi=\ln |y|, \quad l=\frac{e^{\gamma \tau}}{\gamma} .
$$

3.4. Functions. We introduce below the functions $\hat{w}_{0}, \bar{w}_{0}, \bar{U}$, which play important roles in the paper.

- For every $A>0$, we define $\hat{w}_{0}(\eta)$ to be the outer region ansatz

$$
\hat{w}_{0}(\eta):=(n-1)(n-2)\left[1-\left(\frac{\eta}{A}\right)^{-\frac{1}{\gamma}}\right] \text { on } \eta>A,
$$

which is a solution of 2.6).

- For the same $A>0$ as above, we denote by $\bar{w}_{0}(\xi)$ the inner region approximation which the solution of equation

$$
(n-1)\left(\frac{\bar{w}_{\xi \xi}}{\bar{w}}+\frac{n-6}{4} \frac{\bar{w}_{\xi}^{2}}{\bar{w}^{2}}\right)-(n-1)(n-2)+\gamma A \bar{w}_{\xi}=0 .
$$

having asymptotic behavior

$$
\bar{w}_{0}(\xi)=\frac{(n-1)(n-2)}{\gamma A} \xi+0+\frac{(n-1)(n-6)}{4 \gamma A} \frac{1}{\xi}+O\left(\frac{1}{\xi^{2}}\right) .
$$

This solution represents a steady gradient soliton of the flow and it is unique up to translation in $\xi$.

- Finally $\bar{U}$ denotes the representation of $\bar{w}_{0}$ on $\mathbb{R}^{n}$ by the following change of coordinate

$$
|x|^{2} \bar{U}^{1-m}(x)=\bar{w}_{0}(\ln |x|) .
$$

\section{BARRIER CONSTRUCTION IN THE OUTER REGION}

Let us fix parameters $\gamma>0$ and $A>0$ as they appear in the curvature blow up rate of our singularity (2.1). In this section we will construct appropriate super and sub solutions in the outer region $\left(e^{\gamma \tau} \hat{w}(\eta, \tau)\right)^{-1}=o(1), \quad$ as $\tau \rightarrow+\infty$, which will be given by

$$
\left\{(\eta, \tau) \mid \eta \geq A+\xi_{0} e^{-\gamma \tau}, \tau \geq \tau_{0}\right\}
$$

for some $\xi_{0}>0$.

Recall that for a rotationally symmetric solution $u(r, t), r=|x|$ of the conformally flat Yamabe flow 1.2, we perform the cylindrical change of coordinates 1.5 leading to a solution $w(s, t)$ of 1.6$)$. As already seen in section 2, to capture the behavior in the outer region we perform a further change of variables $(2.2)$ leading to a solution $\hat{w}(\eta, \tau)$ of 2.3 . Assuming that near our singularity 2.5 holds, we find that the zero order behavior of $\hat{w}$ near the singularity in the outer region is given by a solution of the ODE 2.6. The general solution of 2.6 is given by 2.7. for a parameter $\kappa>0$. Thus, setting $A:=\kappa^{1 / \gamma}>0$ we define the zero order approximation of $\hat{w}$ in the outer region to be

$$
\hat{w}_{0}(\eta)=(n-1)(n-2)\left[1-\left(\frac{\eta}{A}\right)^{-1 / \gamma}\right] \quad \text { on } \eta>A .
$$

In this section, we are going to construct sub and super solutions of equation 2.3 in the form

$$
\hat{w}(\eta, \tau)=\hat{w}_{0}(\eta)+e^{-2 \gamma \tau}\left(\hat{w}_{1}(\eta)+\theta \hat{w}_{2}(\eta)\right)
$$


for a parameter $\theta \in \mathbb{R}$. To this end, we will choose $\hat{w}_{1}(\eta)$ and $\hat{w}_{2}(\eta)$ to be solutions of a first order linear ODE with different inhomogeneous terms. By setting

$$
f_{1}(\eta):=-(n-1) \frac{\left(\hat{w}_{0}\right)_{\eta \eta}}{\hat{w}_{0}} \text { and } f_{2}(\eta):=-(n-1) \frac{\left(\hat{w}_{0}\right)_{\eta}^{2}}{\hat{w}_{0}^{2}}
$$

we will choose $w_{1}, w_{2}$ to be solutions of the equations

$$
\begin{array}{ll}
\gamma \eta\left(\hat{w}_{1}\right)_{\eta}+(1+2 \gamma) \hat{w}_{1}=f_{1}(\eta) & \text { on } \eta>A \\
\gamma \eta\left(\hat{w}_{2}\right)_{\eta}+(1+2 \gamma) \hat{w}_{2}=f_{2}(\eta) & \text { on } \eta>A .
\end{array}
$$

Plugging $\hat{w}(\eta, \tau)$ given by 4.2 into the equation gives that

$$
\frac{e^{2 \gamma \tau}}{n-1} B[\hat{w}]=\left(\frac{\left(\hat{w}_{0}\right)_{\eta \eta}}{\hat{w}_{0}}+\theta \frac{\left(\hat{w}_{0}\right)_{\eta}^{2}}{\hat{w}_{0}^{2}}\right)-\left(\frac{\hat{w}_{\eta \eta}}{\hat{w}}+\frac{n-6}{4} \frac{\hat{w}_{\eta}^{2}}{\hat{w}^{2}}\right)
$$

where $B[\cdot]$ is given by (2.4) Thus, from the proposed choice of $\hat{w}_{1}, \hat{w}_{2}$ to satisfy 4.4, one expects that $\hat{w}$ is a subsolution of equation 2.3. if $\theta<\frac{n-6}{4}$ and a supersolution if $\theta>\frac{n-6}{4}$, for all parameters $\gamma>0$. The rest of this section is devoted to the justification of this idea which requires a rather delicate calculation. The case of parameters $\gamma \geq 1 / 2$ is shown in Proposition 4.4 below. As we will see in the proof Proposition 4.7 below, in the case of parameters $\gamma<1 / 2$ one needs to add correction term to $\hat{w}$.

Recalling the definition of $\hat{w}_{0}$ in 4.1 and $f_{1}, f_{2}$ in 4.3 we have

$$
\begin{aligned}
& f_{1}(\eta)=+(n-1) \frac{\gamma+1}{\gamma^{2}} \frac{\eta^{-\frac{1}{\gamma}-2}}{A^{-\frac{1}{\gamma}}-\eta^{-\frac{1}{\gamma}}}>0 \\
& f_{2}(\eta)=-(n-1) \frac{1}{\gamma^{2}} \frac{\eta^{-\frac{2}{\gamma}-2}}{\left(A^{-\frac{1}{\gamma}}-\eta^{-\frac{1}{\gamma}}\right)^{2}}<0
\end{aligned}
$$

hence the explicit solutions $w_{i}, i=1,2$ of (4.4) are given by

$$
\hat{w}_{i}(\eta)=\frac{\eta_{0}^{2+\frac{1}{\gamma}} \hat{w}_{i}\left(\eta_{0}\right)}{\eta^{2+\frac{1}{\gamma}}}+\frac{1}{\eta^{2+\frac{1}{\gamma}}} \int_{\eta_{0}}^{\eta} \frac{f_{i}(x)}{\gamma} x^{1+\frac{1}{\gamma}} d x, \quad \eta>A .
$$

We will now fix the functions $\hat{w}_{1}$ and $\hat{w}_{2}$ by fixing their values at a given point $\eta_{0}>A$. While doing so, we will choose $\hat{w}_{2}$ to be a positive function on $\eta>A$. Indeed, since $f_{2}(\eta) \eta^{1+\frac{1}{\gamma}}$ is integrable near $\eta=\infty$, we may choose

$$
\hat{w}_{2}(\eta):=-\frac{1}{\eta^{2+\frac{1}{\gamma}}} \int_{\eta}^{+\infty} \frac{f_{2}(x)}{\gamma} x^{1+\frac{1}{\gamma}} d x>0
$$

that is we choose $\hat{w}_{2}\left(\eta_{0}\right):=-\gamma^{-1} \eta_{0}^{-\left(2+\frac{1}{\gamma}\right)} \int_{\eta_{0}}^{+\infty} f_{2}(x) x^{1+\frac{1}{\gamma}} d x$ in 4.7). For the function $\hat{w}_{1}$ we may choose any value as $\hat{w}_{1}\left(\eta_{0}\right)$, since we do not need to choose it to be positive. Note that by choosing $\hat{w}_{2}$ to be positive, the family of functions $\hat{w}_{1}+\theta \hat{w}_{2}$ is monotone in $\theta \in \mathbb{R}$. To simplify the notation we will simply set from now on

$$
\hat{h}:=\hat{w}_{1}+\theta \hat{w}_{2} .
$$

We will investigate the behavior of the family $\hat{h}(\eta):=\left(\hat{w}_{1}+\theta \hat{w}_{2}\right)(\eta), \theta \in \mathbb{R}$, as $\eta \rightarrow A^{+}$and $\eta \rightarrow+\infty$. We will first see that the behavior near $\eta=A^{+}$is governed by $\hat{w}_{2}$. 
Lemma 4.1. For any linear combination $\hat{h}:=\hat{w}_{1}+\theta \hat{w}_{2}$ of the solutions $\hat{w}_{1}, \hat{w}_{2}$ chosen above we have, we have

$$
\begin{aligned}
& \hat{h}(\eta)=+\frac{\theta}{\gamma A} \frac{n-1}{(\eta-A)}+o\left(\frac{1}{(\eta-A)}\right) \\
& \hat{h}^{\prime}(\eta)=-\frac{\theta}{\gamma A} \frac{n-1}{(\eta-A)^{2}}+o\left(\frac{1}{(\eta-A)^{2}}\right) \\
& \hat{h}^{\prime \prime}(\eta)=+\frac{2 \theta}{\gamma A} \frac{n-1}{(\eta-A)^{3}}+o\left(\frac{1}{(\eta-A)^{3}}\right)
\end{aligned}
$$

as $\eta \rightarrow A^{+}$.

Proof. By 4.7 we have

$$
\hat{h}(\eta)=\frac{\eta_{0}^{2+\frac{1}{\gamma}}\left(\hat{w}_{1}+\theta \hat{w}_{2}\right)\left(\eta_{0}\right)}{\eta^{2+\frac{1}{\gamma}}}+\frac{1}{\eta^{2+\frac{1}{\gamma}}} \int_{\eta_{0}}^{\eta} \frac{\left(f_{1}+\theta f_{2}\right)(x)}{\gamma} x^{1+\frac{1}{\gamma}} d x .
$$

Now, by Taylor's theorem, 4.6 and derivatives of these equations imply the following behavior as $\eta \rightarrow A^{+}$

$$
\left(f_{1}+\theta f_{2}\right)(\eta)=-\frac{\theta(n-1)}{(\gamma-A)^{2}}+O\left((\eta-A)^{-1}\right)
$$

and

$$
\left(f_{1}+\theta f_{2}\right)^{\prime}(\eta)=2 \frac{\theta(n-1)}{(\gamma-A)^{3}}+O\left((\eta-A)^{-2}\right)
$$

In particular we see from the above that $\hat{w}_{2}$ dominates as $\eta \rightarrow A^{+}$and by L'Hôpital's rule on $(\eta-A) \int_{\eta_{0}}^{\eta} \eta^{-1}\left(f_{1}+\theta f_{2}\right)(x) x^{1+\frac{1}{\gamma}} d x$ we obtain

$$
\lim _{\eta \rightarrow A+}(\eta-A) \hat{h}(\eta)=\frac{(n-1) \theta}{\gamma A} .
$$

Similarly, taking derivatives in 4.9$)$ and using the asymptotics for $\left(f_{1}+\theta f_{2}\right)$ and $\left(f_{1}+\theta f_{2}\right)^{\prime}$, we obtain

$$
\lim _{\eta \rightarrow A+}(\eta-A)^{2} \hat{h}^{\prime}(\eta)=-\frac{(n-1) \theta}{\gamma A}, \quad \lim _{\eta \rightarrow A+}(\eta-A)^{3} \hat{h}^{\prime \prime}(\eta)=\frac{2(n-1) \theta}{\gamma A} .
$$

Remark 4.1. Lemma 4.1 shows that $\hat{w}(\eta, \tau)$ defined by 4.2 , which is the ansatz for super and sub solutions, for $\theta>\frac{n-6}{4}$ and $\theta<\frac{n-6}{4}$ respectively, predicts the correct lower order asymptotic behavior in the outer region which is then matched with the inner region where a steady soliton comes in. Indeed, in our previous work CD we showed that a steady soliton of 1.2 satisfies the asymptotics

$$
\bar{w}(\xi)=\frac{(n-1)(n-2)}{\gamma A} \xi+\kappa+\frac{(n-1)(n-6)}{4 \gamma A} \frac{1}{\xi}+o\left(\frac{1}{\xi}\right) .
$$

On the other hand, Lemma 4.1 yields

$$
\hat{w}(\eta, \tau)=\frac{(n-1)(n-2)}{\gamma A}(\eta-A)+e^{-2 \gamma \tau} \frac{(n-1) \theta}{\gamma A} \frac{1}{\eta-A}+O\left((\eta-A)^{2}\right) .
$$

Hence, if we match the inner-outer variables by setting $\eta=A+\xi e^{-\gamma \tau}$, we obtain

$$
e^{\gamma \tau} \hat{w}(\eta, \tau)=\frac{(n-1)(n-2)}{\gamma A} \xi+\frac{(n-1) \theta}{\gamma A} \frac{1}{\xi}+O\left(\left(\xi^{2}+1\right) e^{-\gamma \tau}\right) .
$$


Also, this suggests that in the inner region $\bar{w}$ should be $\bar{w}_{0}$, the translating soliton which satisfies asymptotics 4.10 with $\kappa=0$.

We will next see that behavior of $\left(\hat{w}_{1}+\theta \hat{w}_{2}\right)(\eta)$ as $\eta \rightarrow \infty$ is governed by $\hat{w}_{1}$.

Lemma 4.2. For any linear combination $\hat{h}:=\hat{w}_{1}+\theta \hat{w}_{2}$ of the solutions $\hat{w}_{1}, \hat{w}_{2}$ chosen above we have, we have

$$
\begin{aligned}
& \hat{h}(\eta)=\frac{(n-1)(1+\gamma)}{\gamma^{3}} A^{\frac{1}{\gamma}} \eta^{-\frac{1}{\gamma}-2} \ln \eta+o\left(\eta^{-\frac{1}{\gamma}-2} \ln \eta\right) \\
& \hat{h}^{\prime}(\eta)=-\frac{(n-1)(1+\gamma)(1+2 \gamma)}{\gamma^{4}} A^{\frac{1}{\gamma}} \eta^{-\frac{1}{\gamma}-3} \ln \eta+o\left(\eta^{-\frac{1}{\gamma}-3} \ln \eta\right) \\
& \hat{h}^{\prime \prime}(\eta)=\frac{(n-1)(1+\gamma)(1+2 \gamma)(1+3 \gamma)}{\gamma^{5}} A^{\frac{1}{\gamma}} \eta^{-\frac{1}{\gamma}-4} \ln \eta+o\left(\eta^{-\frac{1}{\gamma}-4} \ln \eta\right)
\end{aligned}
$$

as $\eta \rightarrow \infty$.

Proof. The proof is similar as in Lemma 4.1 if we check the asymptotics

$$
\begin{aligned}
& f_{1}(\eta)=\frac{(n-1)(1+\gamma)}{\gamma^{2}}\left(A^{\frac{1}{\gamma}} \eta^{-\frac{1}{\gamma}-2}+A^{\frac{2}{\gamma}} x^{-\frac{2}{\gamma}-2}\right)+o\left(x^{-\frac{2}{\gamma}-2}\right) \\
& f_{2}(\eta)=-\frac{(n-1)}{\gamma^{2}}\left(A^{\frac{2}{\gamma}} \eta^{-\frac{2}{\gamma}-2}+2 A^{\frac{3}{\gamma}} x^{-\frac{3}{\gamma}-2}\right)+o\left(\eta^{-\frac{3}{\gamma}-2}\right)
\end{aligned}
$$

and corresponding asymptotics for $f_{1}^{\prime}$ and $f_{2}^{\prime}$ as $\eta \rightarrow \infty$.

In the next lemma we give more precise asymptotics which will be used later when we have to add higher order terms to barrier in the case $0<\gamma \leq \frac{1}{2}$. Notice that $\eta^{-\frac{1}{\gamma}-2}$ is a solution of the homogenous equation of (4.4) and we have constants $C$ in the lemma below since we haven't chosen a specific $\hat{w}_{1}$.

Lemma 4.3. For any linear combination $\hat{h}:=\hat{w}_{1}+\theta \hat{w}_{2}$ of the solutions $\hat{w}_{1}, \hat{w}_{2}$ chosen above, we have

$$
\begin{aligned}
\hat{h}(\eta)= & +\frac{(n-1)(1+\gamma)}{\gamma^{3}} A^{\frac{1}{\gamma}} \eta^{-\frac{1}{\gamma}-2} \ln \eta+C \eta^{-\frac{1}{\gamma}-2} \\
& -(n-1) \frac{(n-1)(1+\theta+\gamma)}{\gamma^{2}} A^{\frac{2}{\gamma}} \gamma^{-\frac{2}{\gamma}-2}+o\left(\eta^{-\frac{2}{\gamma}-2}\right) \\
\hat{h}^{\prime}(\eta)= & -\frac{(n-1)(1+\gamma)(1+2 \gamma)}{\gamma^{4}} A^{\frac{1}{\gamma}} \eta^{-\frac{1}{\gamma}-3} \ln \eta \\
& +C^{\prime} \eta^{-\frac{1}{\gamma}-3}+o\left(\eta^{-\frac{2}{\gamma}-2}\right) \\
\hat{h}^{\prime \prime}(\eta)= & +\frac{(n-1)(1+\gamma)(1+2 \gamma)(1+3 \gamma)}{\gamma^{5}} A^{\frac{1}{\gamma}} \eta^{-\frac{1}{\gamma}-4} \ln \eta \\
& +C^{\prime \prime} \eta^{-\frac{1}{\gamma}-4}+o\left(\eta^{-\frac{2}{\gamma}-2}\right)
\end{aligned}
$$

as $\eta \rightarrow+\infty$. Here, $C, C^{\prime}$, and $C^{\prime \prime}$ are constants depending on the choice of $\hat{w}_{1}$.

Proof. Can be shown in the same manner as in Lemma 4.2

We will now show that $\hat{w}(\eta, \tau)$ given by $(4.2)$ is a sub or super - solution of equation (2.3) in the appropriate regions. We will first deal with the case of parameters $\gamma>1 / 2$. The case $\gamma \leq 1 / 2$ is more delicate and will be considered later. 
Proposition 4.4. For any $\gamma>1 / 2$ and given $\theta \neq \frac{n-6}{4}$, there exist $\tau_{0} \in \mathbb{R}$ and $\xi_{0}>0$ depending on $n, A, \gamma$ and $\theta$ such that the function

$$
\hat{w}(\eta, \tau):=\hat{w}_{0}(\eta)+e^{-2 \gamma \tau}\left(\hat{w}_{1}(\eta)+\theta \hat{w}_{2}(\eta)\right)
$$

is a subsolution of equation (2.3) if $\theta<\frac{n-6}{4}$ or a supersolution if $\theta>\frac{n-6}{4}$, respectively, in the region

$$
\left\{(\eta, \tau) \mid \eta \geq A+\xi_{0} e^{-\gamma \tau}, \tau \geq \tau_{0}\right\} .
$$

Proof of Proposition 4.4. As before, let us denote by

$$
\hat{h}:=\hat{w}_{1}+\theta \hat{w}_{2} .
$$

We need to show that

$$
B[\hat{w}]<0, \quad \text { if } \theta<\frac{n-6}{4} \quad \text { or } \quad B[\hat{w}]>0, \quad \text { if } \theta>\frac{n-6}{4}
$$

holds in the region

Proposition 4.4 follows from the the two claims below

Claim 4.5. For any $\gamma>0$ and given $\theta \neq \frac{n-6}{4}$, there exist $\xi_{0}>0$ and $\delta>0$ such that $\bar{w}(\eta, \tau)$ is a subsolution of equation 2.3) if $\theta<\frac{n-6}{4}$ or a supersolution if $\theta>\frac{n-6}{4}$, respectively, in the region

$$
\left\{(\eta, \tau) \mid A+\xi_{0} e^{-\gamma \tau} \leq \eta<A+\delta, \tau \in \mathbb{R}\right\} .
$$

Proof of Claim 4.5. By Lemma 4.1, we may find $\kappa=\kappa(n, A, \gamma)>0$ such that

$$
|\hat{h}|(\eta-A),\left|\hat{h}^{\prime}\right|(\eta-A)^{2},\left|\hat{h}^{\prime \prime}\right|(\eta-A)^{3}<\kappa|\theta|
$$

holds on the region $A<\eta<A+1$. Moreover, by Taylor's theorem we may choose the constant $\kappa$ so that

$$
\left|\hat{w}_{0}(\eta)-\frac{(n-1)(n-2)}{\gamma A}(\eta-A)\right|<\kappa(\eta-A)^{2}
$$

and

$$
\left|\hat{w}_{0}^{\prime}(\eta)-\frac{(n-1)(n-2)}{\gamma A}\right|<\kappa(\eta-A) \quad \text { and } \quad\left|\hat{w}_{0}^{\prime \prime}(\eta)\right|<\kappa
$$

hold. Using these, we get

$$
\begin{aligned}
\left|\hat{w}-\frac{(n-1)(n-2)}{\gamma A}(\eta-A)\right| & =\left|\hat{w}_{0}-\frac{(n-1)(n-2)}{\gamma A}(\eta-A)+e^{-2 \gamma \tau} \hat{h}\right| \\
& \leq \kappa(\eta-A)^{2}+\kappa|\theta| e^{-2 \gamma \tau} \frac{1}{(\eta-A)} \\
& =\left(\kappa(\eta-A)+\frac{\kappa|\theta|}{\left((\eta-A) e^{\gamma \tau}\right)^{2}}\right)(\eta-A) \\
& \leq \kappa\left(\delta+\frac{|\theta|}{\xi_{0}^{2}}\right)(\eta-A) .
\end{aligned}
$$

Hence by restricting to $0<\delta<1$ small and $\xi_{0}>0$ large, we may assume that

$$
\hat{w}>\frac{(n-1)(n-2)}{2 \gamma A}(\eta-A)>0 .
$$


Using the above we find that in the considered region we have

$$
\begin{aligned}
\left|\frac{\hat{w}_{\eta \eta}}{\hat{w}}\right| \leq \frac{\left|\hat{w}_{0}^{\prime \prime}\right|+\left|e^{-2 \gamma \tau} \hat{h}^{\prime \prime}\right|}{\hat{w}} & \leq \frac{1}{(\eta-A)} \frac{2 \gamma A}{(n-1)(n-2)}\left(\kappa+\frac{|\theta| \kappa}{(\eta-A)^{3} e^{2 \gamma \tau}}\right) \\
& \leq \frac{\kappa}{(\eta-A)^{2}} \frac{2 \gamma A}{(n-1)(n-2)}\left(\delta+\frac{|\theta|}{\xi_{0}^{2}}\right)
\end{aligned}
$$

and

$$
\begin{aligned}
\left|\frac{\hat{w}_{\eta}^{2}}{\hat{w}^{2}}\right| & =\frac{\left|\hat{w}_{0}^{\prime}\right|^{2}\left|1+e^{-2 \gamma \tau} \hat{h}^{\prime} / \hat{w}_{0}^{\prime}\right|^{2}}{\hat{w}^{2}} \\
& \leq \frac{1}{(\eta-A)^{2}}\left|\frac{\hat{w}_{0}^{\prime 2}}{\hat{w}^{2}(\eta-A)^{-2}}\left(1+\frac{|\theta| \kappa}{(\eta-A)^{2} e^{2 \gamma \tau} \hat{w}_{0}^{\prime}}\right)^{2}\right| \\
& \leq \frac{1}{(\eta-A)^{2}}\left(\frac{\frac{(n-1)(n-2)}{\gamma A}+\kappa \delta}{\frac{(n-1)(n-2)}{\gamma A}-\kappa\left(\delta+\frac{|\theta|}{\xi_{0}^{2}}\right)}\right)^{2}\left(1+\frac{|\theta| \kappa}{\xi_{0}^{2}\left(\frac{(n-1)(n-2)}{\gamma A}-\kappa \delta\right)}\right)^{2} .
\end{aligned}
$$

Similarly, we estimate from below

$$
\left|\frac{\hat{w}_{\eta}^{2}}{\hat{w}^{2}}\right| \geq \frac{1}{(\eta-A)^{2}}\left(\frac{\frac{(n-1)(n-2)}{\gamma A}-\kappa \delta}{\frac{(n-1)(n-2)}{\gamma A}+\kappa\left(\delta+\frac{|\theta|}{\xi_{0}^{2}}\right)}\right)\left(1-\frac{|\theta| \kappa}{\xi_{0}^{2}\left(\frac{(n-1)(n-2)}{\gamma A}-\kappa \delta\right)}\right)^{2} .
$$

Thus, for a fixed $\epsilon>0$ to be determined later, we may find small $\delta>0$ and large $\xi_{0}>0$ so that all

$$
\left|\frac{\hat{w}_{\eta}^{2}}{\hat{w}^{2}}-\frac{1}{(\eta-A)^{2}}\right|,\left|\frac{\hat{w}_{0}^{\prime 2}}{\hat{w}_{0}^{2}}-\frac{1}{(\eta-A)^{2}}\right|,\left|\frac{\hat{w}_{\eta \eta}}{\hat{w}}\right|,\left|\frac{\hat{w}_{0}^{\prime \prime}}{\hat{w}_{0}}\right| \leq \frac{\epsilon}{(\eta-A)^{2}} .
$$

Recalling the formula for $B[\hat{w}]$ in 4.5 and using the triangle inequality successively we obtain

$$
\left|\frac{e^{2 \gamma \tau} B[\hat{w}]}{(n-1)}-\left(\theta-\frac{n-6}{4}\right) \frac{1}{(\eta-A)^{2}}\right| \leq \epsilon \frac{|\theta|+\left|\frac{n-6}{4}\right|+2}{(\eta-A)^{2}} .
$$

Finally, by choosing $\epsilon:=\frac{1}{2}\left|\theta-\frac{n-6}{4}\right|\left(|\theta|+\left|\frac{n-6}{4}\right|+2\right)^{-1}$ we conclude that 4.5 holds, finishing the proof of the claim.

We will next proceed in our next claim which holds for $\gamma>1 / 2$.

Claim 4.6. For $\gamma>1 / 2$ and given $\theta \neq \frac{n-6}{4}$ and $\delta>0$, there is $\tau_{0}=\tau_{0}(\theta, \delta, \gamma)$ such that such that $\hat{w}(\eta, \tau)$ is a subsolution of equation 2.3) if $\theta<\frac{n-6}{4}$ or a supersolution if $\theta>\frac{n-6}{4}$ on the set

$$
\left\{(\eta, \tau) \mid \eta>A+\delta, \tau \geq \tau_{0}\right\}
$$

Proof of Claim 4.6. For a given $\delta_{0}>0$, Lemma 4.2 and the asymptotic behavior of $\hat{w}_{0}, \hat{w}_{0}^{\prime}$ and $\hat{w}_{0}^{\prime \prime}$ can be used to find a constant $\kappa>0$ such that

$$
\left|\frac{\hat{h}}{w_{0}}\right|,\left|\frac{\hat{h}^{\prime}}{w_{0}^{\prime}}\right|,\left|\frac{\hat{h}^{\prime \prime}}{w_{0}^{\prime \prime}}\right|<\kappa \frac{\ln (1+\eta)}{\eta^{2}}<\frac{\kappa}{A+\delta_{0}} \quad \text { on } \eta>A+\delta_{0} .
$$

Thus, we may start with some large $\tau_{0}$ such that $\hat{w}, \hat{w}_{\eta}, \hat{w}_{\eta \eta}$ have same the sign as $\hat{w}_{0}, \hat{w}_{0}^{\prime}, \hat{w}_{0}^{\prime \prime}$ respectively 
on $\eta>A+\delta$ and $\tau>\tau_{0}$. In particular, they are nonzero. Using the formula for $B[\hat{w}]$ in 4.5 , we write

$$
\frac{e^{2 \gamma \tau}}{(n-1)} B[\hat{w}]=\left(\frac{\hat{w}_{0}^{\prime \prime}}{\hat{w}_{0}}-\frac{\hat{w}_{\eta \eta}}{\hat{w}}\right)+\frac{n-6}{4}\left(\frac{\hat{w}_{0}^{\prime 2}}{\hat{w}^{2}}-\frac{\hat{w}_{\eta}^{2}}{\hat{w}^{2}}\right)+\left(\theta-\frac{n-6}{4}\right) \frac{\hat{w}_{0}^{\prime 2}}{\hat{w}_{0}^{2}} .
$$

We will show that the first two terms become arbitrarily small in comparison with the last term, for $\tau_{0} \gg 1$ (and hence $\tau \gg 1$ ). Indeed, we have

$$
\left|\frac{\hat{w}_{\eta \eta}}{\hat{w}}-\frac{\hat{w}_{0}^{\prime \prime}}{\hat{w}_{0}}\right|=e^{-2 \gamma \tau}\left|\frac{\hat{w}_{0}^{\prime \prime}}{\hat{w}_{0}}\right|\left|\frac{\left(\hat{h}^{\prime \prime} / \hat{w}_{0}^{\prime \prime}\right)-\left(\hat{h} / \hat{w}_{0}\right)}{1+e^{-2 \gamma \tau}\left(\hat{h} / \hat{w}_{0}\right)}\right|
$$

and

$$
\left|\frac{\hat{w}_{\eta}^{2}}{\hat{w}^{2}}-\frac{\hat{w}_{0}^{\prime 2}}{\hat{w}_{0}^{2}}\right|=e^{-2 \gamma \tau} \frac{\hat{w}_{0}^{\prime 2}}{\hat{w}_{0}^{2}} \frac{\left|2\left(\left(\hat{h}^{\prime} / \hat{w}_{0}^{\prime}\right)-\left(\hat{h} / \hat{w}_{0}\right)\right)+e^{-2 \gamma \tau}\left(\left(\hat{h}^{\prime} / \hat{w}_{0}^{\prime}\right)^{2}-\left(\hat{h} / \hat{w}_{0}\right)^{2}\right)\right|}{\left(1+e^{-2 \gamma \tau}\left(\hat{h} / \hat{w}_{0}\right)\right)^{2}} .
$$

Thus, the asymptotics in Lemma 4.3 imply that by choosing $\tau_{0} \gg 1$ we have

$$
\begin{aligned}
& \left|\frac{\hat{w}_{\eta \eta}}{\hat{w}}-\frac{\hat{w}_{0}^{\prime \prime}}{\hat{w}_{0}}\right| \leq 10 e^{-2 \gamma \tau} \kappa\left|\frac{\hat{w}_{0}^{\prime \prime}}{\hat{w}_{0}}\right| \frac{\ln (1+\eta)}{\eta^{2}} \\
& \left|\frac{\hat{w}_{\eta}^{2}}{\hat{w}^{2}}-\frac{\hat{w}_{0}^{\prime 2}}{\hat{w}_{0}^{2}}\right| \leq 10 e^{-2 \gamma \tau} \kappa\left(\frac{\hat{w}_{0}^{\prime 2}}{\hat{w}_{0}^{2}}\right) \frac{\ln (1+\eta)}{\eta^{2}} .
\end{aligned}
$$

for all $\tau>\tau_{0}$. In the case $\gamma>\frac{1}{2}$, by $\frac{\ln \eta}{\eta^{2}}, e^{-2 \gamma \tau}$ terms in the previous estimate and asymptotics (4), we can make $\tau_{0}$ large and conclude

$$
\left|\left(\frac{\hat{w}_{0}^{\prime \prime}}{\hat{w}_{0}}-\frac{\hat{w}_{\eta \eta}}{\hat{w}}\right)+\frac{n-6}{4}\left(\frac{\hat{w}_{0}^{\prime 2}}{\hat{w}^{2}}-\frac{\hat{w}_{\eta}^{2}}{\hat{w}^{2}}\right)\right| \leq \frac{1}{2}\left|\theta-\frac{n-6}{4}\right| \frac{\hat{w}_{0}^{\prime 2}}{\hat{w}_{0}^{2}}
$$

on the considered region. This proves that for $\tau \geq \tau_{0} \gg 1$, 4.11 holds, finishing the proof of the claim.

To finish the proof of the proposition, for any given $\gamma>1 / 2$ and $\theta \neq \frac{n-6}{4}$, Claim 4.5 implies that there exists $\delta>0$ such that such that 4.11 holds in the region $A+\xi_{0} e^{-\gamma \tau} \leq \eta<A+\delta$. In addition, by Claim 4.6 there exists $\tau_{0}=\tau_{0}(\theta, \delta, \gamma)$ such that 4.11 holds in the region $\eta>A+\delta, \tau \geq \tau_{0}$. We conclude that (4.11) holds in the whole outer region $\eta>A+\xi_{0} e^{-\gamma \tau}$ for $\tau \geq \tau_{0}$ finishing the proof of the proposition.

In the case $0<\gamma \leq 1 / 2$, we need to add a higher order correction term in our barrier. For integers $k \geq 2$ and $0 \leq l \leq k$, we define the functions

$$
v_{k, l}(\eta):=\eta^{-2 k-\frac{1}{\gamma}}(\ln \eta)^{l}, \quad \eta>1 .
$$

They satisfy the following relation

$$
(1+2 k \gamma) v_{k, l}+\gamma \eta v_{k, l}^{\prime}= \begin{cases}\gamma l v_{k, l-1} & \text { if } l>0 \\ 0 & \text { if } l=0\end{cases}
$$

and to simplify the notation we also set $v_{k,-1}(\eta)=0$ and $v_{k,-2}(\eta)=0$. We will show the following. 
Proposition 4.7. For any $0<\gamma \leq 1 / 2$ and given $\theta \neq \frac{n-6}{4}$, there exist $\tau_{0} \in \mathbb{R}$, $\xi_{0}>0$, integer $N \geq 2$ and coefficients $\left\{c_{k, l}\right\}_{2 \leq k \leq N, 0 \leq l \leq k}$ such that the function

$$
\hat{w}(\eta, \tau):=\hat{w}_{0}(\eta)+e^{-2 \gamma \tau}\left(\hat{w}_{1}(\eta)+\theta \hat{w}_{2}(\eta)\right)+\Sigma_{k=2}^{N} e^{-2 k \gamma \tau} \Sigma_{l=0}^{k} c_{k, l} v_{k, l}(\eta)
$$

is a subsolution of equation (2.3) if $\theta<\frac{n-6}{4}$ or a supersolution if $\theta>\frac{n-6}{4}$, in the region

$$
\left\{(\eta, \tau) \mid \eta \geq A+\xi_{0} e^{-\gamma \tau}, \tau \geq \tau_{0}\right\}
$$

Proof of Proposition 4.7. For the given $0<\gamma \leq 1 / 2$, let $N$ denote the smallest integer making $\gamma>1 /(2 N)$, namely $N:=[1 /(2 \gamma)]+1$. The next claim corresponds to Claim 4.6 for this case.

Claim 4.8. For any $0<\gamma \leq 1 / 2$ and any given choice of $\left\{c_{k, 0}\right\}_{2 \leq k \leq N}$, there are coefficients $\left\{c_{k, l}\right\}_{2 \leq k \leq N, 0 \leq l \leq k}$ so that for any given $\delta>0$ and $\theta \neq \frac{n-6}{4}$, the function

$$
\hat{w}(\eta, \tau)=\hat{w}_{0}(\eta)+e^{-2 \gamma \tau}\left(\hat{w}_{1}(\eta)+\theta \hat{w}_{2}(\eta)\right)+\sum_{k=2}^{N} e^{-2 k \gamma \tau} \sum_{l=0}^{k} c_{k, l} v_{k, l}(\eta)
$$

is a subsolution of equation 2.3 if $\theta<\frac{n-6}{4}$ or a supersolution if $\theta>\frac{n-6}{4}$ on the set

$$
\left\{(\eta, \tau) \mid \eta>A+\delta, \tau \geq \tau_{0}\right\}
$$

where $\tau_{0}=\tau_{0}(\gamma, \theta, \delta) \gg 1$

Proof of Claim 4.8. Let us assume $\theta<\frac{n-6}{4}$ because the other case follows similarly. Suppose $\hat{w}$ is of the form of (4.8). We will choose the coefficients $c_{k, l}$ later.

We split the operator $B[\cdot]$ given by (2.4) into linear and nonlinear parts, that is we write

$$
B[\hat{w}]=I_{1}[\hat{w}]+(n-1)(n-2)-I_{2}[\hat{w}] .
$$

where

$$
I_{1}[\hat{w}]:=\partial_{\tau} \hat{w}-\gamma \eta \hat{w}_{\eta}-\hat{w}, \quad I_{2}[\hat{w}]:=(n-1)\left(\frac{\hat{w}_{\eta \eta}}{\hat{w}}+\frac{n-6}{4} \frac{\hat{w}_{\eta}^{2}}{\hat{w}^{2}}\right) e^{-2 \gamma \tau}
$$

Then, using (4.3), 4.4 and 4.16) we find

$$
\begin{aligned}
I_{1}[\hat{w}]= & I_{1}\left[\hat{w}_{0}\right]+I_{1}\left[\hat{h} e^{-2 \gamma \tau}\right]+\Sigma_{k=2}^{N} \Sigma_{l=0}^{k} c_{k, l} I_{1}\left[v_{k, l} e^{-2 k \gamma \tau}\right] \\
= & -(n-1)(n-2)+(n-1)\left(\frac{\left(\hat{w}_{0}\right)_{\eta \eta}}{\hat{w}_{0}}+\theta \frac{\left(\hat{w}_{0}\right)_{\eta}^{2}}{\hat{w}_{0}^{2}}\right) e^{-2 \gamma \tau} \\
& -\Sigma_{k=2}^{N} e^{-2 k \gamma \tau} \Sigma_{l=1}^{k} \gamma l c_{k, l} v_{k, l-1} .
\end{aligned}
$$


Meanwhile, using the asymptotics of $\hat{w}_{0}, \hat{w}_{1}+\theta \hat{w}_{2}, v_{k, l}$ and their derivatives

$$
\begin{aligned}
I_{2}[\hat{w}] & =(n-1)\left(\frac{\hat{w}_{\eta \eta}}{\hat{w}}+\frac{n-6}{4} \frac{\left(\hat{w}_{0}\right)_{\eta}^{2}}{\hat{w}_{0}^{2}}+o\left(\eta^{-2-\frac{2}{\gamma}}\right) e^{-2 \gamma \tau}\right) e^{-2 \gamma \tau} \\
= & (n-1)\left(\frac{\hat{w}_{\eta \eta}}{\hat{w}_{0}}+\frac{n-6}{4} \frac{\left(\hat{w}_{0}\right)_{\eta}^{2}}{\hat{w}_{0}^{2}}+o\left(\eta^{-2-\frac{2}{\gamma}}\right) e^{-2 \gamma \tau}\right) e^{-2 \gamma \tau} \\
= & (n-1)\left(\frac{\left(\hat{w}_{0}\right)_{\eta \eta}}{\hat{w}_{0}}+\frac{n-6}{4} \frac{\left(\hat{w}_{0}\right)_{\eta}^{2}}{\hat{w}_{0}^{2}}\right) e^{-2 \gamma \tau} \\
& +(n-1) \frac{\hat{h}_{\eta \eta} e^{-4 \gamma \tau}+\sum_{k=2}^{N} e^{-2(k+1) \gamma \tau} \sum_{l=0}^{k} c_{k, l}\left(v_{k, l}\right)_{\eta \eta}}{\hat{w}_{0}}+o\left(\eta^{-2-\frac{2}{\gamma}}\right) e^{-4 \gamma \tau} \\
= & (n-1)\left(\frac{\left(\hat{w}_{0}\right)_{\eta \eta}}{\hat{w}_{0}}+\frac{n-6}{4} \frac{\left(\hat{w}_{0}\right)_{\eta}^{2}}{\hat{w}_{0}^{2}}\right) e^{-2 \gamma \tau} \\
& +\frac{\hat{h}_{\eta \eta} e^{-4 \gamma \tau}+\Sigma_{k=2}^{N-1} e^{-2(k+1) \gamma \tau} \sum_{l=0}^{k} c_{k, l}\left(v_{k, l}\right)_{\eta \eta}}{(n-2)}+o\left(\eta^{-2-\frac{2}{\gamma}}\right) e^{-4 \gamma \tau}
\end{aligned}
$$

In the last line we used that $2 N>1 / \gamma$. Also, $g(\eta, \tau)=o\left(\eta^{-2-\frac{2}{\gamma}}\right)$ means that $\sup _{\eta>\eta^{\prime}, \tau>\tau^{\prime}} \eta^{2+\frac{2}{\gamma}} g(\eta, \tau) \rightarrow 0$, as $\eta^{\prime} \rightarrow \infty$ for any fixed $\tau^{\prime}$.

Combining the above computations yields

$$
\begin{aligned}
B[\hat{w}]= & (n-1)\left(\theta-\frac{n-6}{4}\right) \frac{\left(\hat{w}_{0}\right)_{\eta}^{2}}{\hat{w}_{0}^{2}} e^{-2 \gamma \tau}-\Sigma_{k=2}^{N} e^{-2 k \gamma \tau} \sum_{l=1}^{k} \gamma l c_{k, l} v_{k, l-1} \\
& -\frac{\hat{h}_{\eta \eta} e^{-4 \gamma \tau}+\Sigma_{k=2}^{N-1} e^{-2(k+1) \gamma \tau} \Sigma_{l=0}^{k} c_{k, l}\left(v_{k, l}\right)_{\eta \eta}}{(n-2)}+o\left(\eta^{-2-\frac{2}{\gamma}}\right) e^{-4 \gamma \tau} \\
= & (n-1)\left(\theta-\frac{n-6}{4}\right) \frac{\left(\hat{w}_{0}\right)_{\eta}^{2}}{\hat{w}_{0}^{2}} e^{-2 \gamma \tau}-\left(\frac{h_{\eta \eta}}{n-2}+\Sigma_{l=1}^{2} \gamma l c_{2, l} v_{2, l-1}\right) e^{-4 \gamma \tau} \\
& -\Sigma_{k=3}^{N}\left(\frac{1}{n-2} \Sigma_{l=1}^{k} c_{k-1, l-1}\left(v_{k-1, l-1}\right)_{\eta \eta}+\Sigma_{l=1}^{k} \gamma l c_{k, l} v_{k, l-1}\right) e^{-2 k \gamma \tau} \\
& +o\left(\eta^{-2-\frac{2}{\gamma}}\right) e^{-4 \gamma \tau} .
\end{aligned}
$$

Let us remark that $\left(v_{k-1, l-1}\right)_{\eta \eta}$ can be written as a linear combination of $\left\{v_{k, l-1}, v_{k, l-2}, v_{k, l-3}\right\}$. Hence for any given $\left\{c_{k, 0}\right\}_{2 \leq k \leq N}$, there is a unique choice $\left\{c_{k, j}\right\}_{2 \leq k \leq N}, 1 \leq l \leq k$ such that

$$
B[\hat{w}]=(n-1)\left(\theta-\frac{n-6}{4}\right) \frac{\left(\hat{w}_{0}\right)_{\eta}^{2}}{\hat{w}_{0}^{2}} e^{-2 \gamma \tau}+o\left(\eta^{-2-\frac{2}{\gamma}}\right) e^{-4 \gamma \tau} .
$$

Here we also used the asymptotic expansion of $h_{\eta \eta}$ as $\eta \rightarrow \infty$, namely

$$
h_{\eta \eta}=(n-1) A^{\frac{1}{\gamma}} \frac{(1+\gamma)(1+2 \gamma)(1+3 \gamma)}{\gamma^{5}} v_{2,1}+C^{\prime \prime} v_{2,0}+o\left(\eta^{-2-\frac{2}{\gamma}}\right)
$$

which has been shown in Lemma 4.3. Finally, we may find a large $\tau_{0}=\tau_{0}(\delta, \gamma, \theta)$ such that $B[\hat{w}]<0$ on the region $\eta>A+\delta$ for $\tau \geq \tau_{0}$. This finishes the proof of our claim.

As we fixed $\hat{w}_{1}$ and $\hat{w}_{2}$ in the proof of Proposition 4.4 from now on let us fix $c_{k, l}$ so that Claim 4.8 holds, by choosing $c_{k, 0}=0$. Next, we give the analogue of Claim 4.5 in this case. 
Claim 4.9. For given $0<\gamma \leq 1 / 2, \theta \neq \frac{n-6}{4}$, there exist $\xi_{0}>0$ and $\delta>0$ such that

$$
\hat{w}(\eta, \tau)=\hat{w}_{0}(\eta)+e^{-2 \gamma \tau}\left(\hat{w}_{1}(\eta)+\theta \hat{w}_{2}(\eta)\right)+\Sigma_{k=2}^{N} e^{-2 k \gamma \tau} \sum_{l=0}^{k} c_{k, l} v_{k, l}(\eta)
$$

is is a subsolution of equation 2.3) if $\theta<\frac{n-6}{4}$ and a supersolution if $\theta>\frac{n-6}{4}$ on the region

$$
\left\{(\eta, \tau) \mid A+\xi_{0} e^{-\gamma \tau}<\eta<A+\delta, \tau>0\right\} .
$$

Proof of Claim 4.9. By rewriting $\hat{w}(\eta, \tau)=\hat{w}_{0}(\eta)+e^{-2 \gamma \tau} \hat{h}(\eta, \tau)$, we have the same estimate of Proposition 4.1 and the proof is actually the same as of Claim 4.5 .

The proof of the Proposition 4.7 now readily follows by combining claims 4.8 and 4.9 Let us fix $0<\gamma \leq 1 / 2$ and $\theta \neq \frac{n-6}{4}$. Let $c_{k, l}$ be coefficients with $c_{k, 0}=0$ be so that Claim 4.8 holds. For that choice of $c_{k, l}$, Claim 4.9 gives the existence of $\xi_{0}>0$ and $\delta>0$ so that $\hat{w}$ is a subsolution (supersolution) in the region $A+\xi_{0} e^{-\gamma \tau}<\eta<A+\delta, \tau>0$. By Claim 4.8 there exists $\tau_{0}=\tau_{0}(\gamma, \theta, \delta)$ such that $\hat{w}$ is a subsolution (supersolution) in the region $\eta>A+\delta, \tau \geq \tau_{0}$. We conclude that $\hat{w}$ is a subsolution (supersolution) in the region $\eta>A+\xi_{0} e^{-\gamma \tau}, \tau \geq \tau_{0}$. Since $\delta=\delta(\gamma, \theta)$ we also have that $\tau_{0}=\tau_{0}(\gamma, \theta)$.

\section{BARRIER CONSTRUCTION IN THE INNER REGION}

We will now construct the appropriate barrier in the inner region which is the region where

$$
e^{\gamma \tau} \hat{w}(\eta, \tau)=O(1), \quad \text { as } \tau \rightarrow+\infty .
$$

In this region we define $\bar{w}(\xi, \tau)$ as in 2.11, that is we set $\bar{w}(\xi, \tau)=e^{\gamma \tau} \hat{w}(\eta, \tau)$, $\xi=(\eta-A) e^{\gamma \tau}$. We have seen in section 2 that $\bar{w}(\xi, \tau)$ satisfies the equation $I[\bar{w}]=0$ with $I[\cdot]$ given by $(2.13)$. Let us assume that in this region the first term in 2.13 having $e^{-\gamma \tau}$ becomes negligible as $\tau \rightarrow \infty$. Then, we expect that the solution $\bar{w}_{0}(\xi)$ of equation

$$
\frac{\left(\bar{w}_{0}\right)_{\xi \xi}}{\bar{w}}+\frac{n-6}{4} \frac{\left(\bar{w}_{0}\right)_{\xi}^{2}}{\bar{w}^{2}}-(n-1)(n-2)+\gamma A\left(\bar{w}_{0}\right)_{\xi}=0
$$

is the leading order term for $\bar{w}(\xi, \tau)$ in this region. We are going to find super and sub solutions $\bar{w}^{+}$and $\bar{w}^{-}$, respectively in the following form

$$
\begin{aligned}
& \bar{w}^{+}(\xi, \tau)=\frac{1}{1+\epsilon} \bar{w}_{0}\left(\xi+C_{1}(\tau)\right) \\
& \bar{w}^{-}(\xi, \tau)=\frac{1}{1-\epsilon} \bar{w}_{0}\left(\xi+C_{2}(\tau)\right) .
\end{aligned}
$$

Here, $\epsilon>0$ is a small constant and $C_{1}(\tau)$ and $C_{2}(\tau)$ are smooth functions of $\tau$. Both will be chosen later and will depend on $\xi_{0}$ which appears in the construction of our barriers in the outer region. As we will see below, the construction is rather straightforward.

If we plug these into $I[\cdot]$, we get

$$
\begin{aligned}
& I\left[\bar{w}^{+}\right]=+\epsilon \bar{w}_{\xi}^{+}+e^{-\gamma \tau}\left(C_{1}^{\prime}(\tau) \bar{w}_{\xi}^{+}-(1+\gamma) \bar{w}^{+}\right) \\
& I\left[\bar{w}^{-}\right]=-\epsilon \bar{w}_{\xi}^{-}+e^{-\gamma \tau}\left(C_{2}^{\prime}(\tau) \bar{w}_{\xi}^{+}-(1+\gamma) \bar{w}^{-}\right) .
\end{aligned}
$$

We will next show the following. 
Proposition 5.1. Let $0<\epsilon<1$ and $\tau_{0} \in \mathbb{R}$. If $\left|C_{1}^{\prime}(\tau)\right|,\left|C_{2}^{\prime}(\tau)\right| \leq M$ on $\tau \geq \tau_{0}$, then $\xi_{1}$ there exist $\tau_{1}=\tau_{1}\left(\epsilon, M, \xi_{1}\right) \geq \tau_{0}$ such that $\bar{w}^{+}$or $\bar{w}^{-}$are super or sub solutions of equation 2.9) respectively, in the region $(\xi, \tau) \in\left(-\infty, \xi_{1}\right) \times\left(\tau_{1}, \infty\right)$.

Proof. For any two functions $f(s), g(s)$ we use the notation

$$
f(s) \sim g(s), \text { as } s \rightarrow \infty \quad \text { iff } \quad c<\left|\frac{f(s)}{g(s)}\right|<C, \text { for } s \gg 1
$$

for some fixed constants $c>0, C<+\infty$.

In this proof we will use the asymptotics for $\bar{w}_{0}(s)$ and $\bar{w}_{0}^{\prime}(s)$, as $s \rightarrow \infty$, which were shown in Proposition 2.1 in [CD] or [DS2, [H] . Since $\bar{w}_{0}(s)=e^{2 s} \bar{U}^{1-m}\left(e^{s}\right)$ and $\bar{U}^{1-m}(|x|) \delta_{i j}$ is a smooth radial metric on $\mathbb{R}^{n}$, we have

$$
\bar{w}_{0} \sim\left(\bar{w}_{0}\right)_{s} \sim e^{2 s}, \quad \text { as } s \rightarrow \infty .
$$

Moreover since $\bar{w}_{0} \sim s,\left(\bar{w}_{0}\right)_{s} \sim 1$, as $s \rightarrow \infty$, it is clear that there is some $\tau_{2}$ and $c$ so that

$$
e^{-\gamma \tau}(1+\gamma) \bar{w}_{0}(s)<\frac{\epsilon}{2}\left(\bar{w}_{0}\right)_{s}(s), \quad(s, \tau) \in\left(-\infty, c e^{\gamma \tau}\right) \times\left(\tau_{2}, \infty\right) .
$$

Now given $\xi_{1}$ and $C_{1}(\tau)$ and $C_{2}(\tau)$ satisfying the conditions in our proposition, we can find some $\tau_{1}>\max \left(\tau_{0}, \tau_{2}\right)$ such that

$$
\xi_{1}+C_{i}(\tau)<c e^{\gamma \tau} \text { and } \quad\left|e^{-\gamma \tau} C_{i}^{\prime}(\tau)\right|<\frac{\epsilon}{2}, \quad \text { for } \tau>\tau_{1} .
$$

The last two formulas and the fact that $w_{0}>0,\left(w_{0}\right)_{s}>0$, imply that $I\left[\bar{w}^{+}\right]>0$ and $I\left[\bar{w}^{-}\right]<0$ on $(\xi, \tau) \in\left(-\infty, \xi_{1}\right) \times\left(\tau_{1}, \infty\right)$, as claimed.

\section{Construction of Super And SUb-SOlutions}

In this section we will combine the results from Sections 4 and 5 to construct a family of super-solutions $w_{\epsilon}^{+}$and sub-solutions $w_{\epsilon}^{-}$of equation (1.6) which is equivalent to the conformally flat Yamabe flow 1.2 under rotational symmetry and after the cylindrical change of variables 1.5 . This will give a family of rotationally symmetric super and sub solutions of equation 1.2 which we will then be used in the next section to analyze the type II blow up behavior of any solution $u(x, t)$ of 1.2 which satisfies the assumptions of Theorem 6.4.

We begin by fixing

$$
\gamma>0, \quad A>0, \quad \theta^{+}>\frac{n-6}{4}, \quad \theta^{-}<\frac{n-6}{4} .
$$

For these choices of parameters and following the results in Section 4 , we define the super and sub-solutions $\hat{w}^{+}$and $\hat{w}^{-}$corresponding to $\theta^{+}$and $\theta^{-}$respectively in the outer region $\eta>A+\xi_{0} e^{-\gamma \tau}$ separately for different ranges of $\gamma$ : for $\gamma>1 / 2$ we set

$$
\hat{w}^{ \pm}(\eta, \tau):=\hat{w}_{0}(\eta)+e^{-2 \gamma \tau}\left(\hat{w}_{1}(\eta)+\theta^{ \pm} \hat{w}_{2}(\eta)\right)
$$

while for $0<\gamma \leq 1 / 2$, we add the extra correction term setting

$$
\hat{w}^{ \pm}(\eta, \tau):=\hat{w}_{0}(\eta)+e^{-2 \gamma \tau}\left(\hat{w}_{1}(\eta)+\theta^{ \pm} \hat{w}_{2}(\eta)\right)+\Sigma_{k=2}^{N} \sum_{l=0}^{k} c_{k, l} e^{-2 k \gamma \tau} v_{k, l}(\eta) .
$$

Propositions 4.4 and 4.7 , show that there exist $\tau_{0}$ and $\xi_{0}>0$, such that $\hat{w}^{+}$and $\hat{w}^{-}$ are super and sub solutions, respectively on the region $(\eta, \tau) \in\left(A+\xi_{0} e^{-\gamma \tau}, \infty\right) \times$ 
$\left[\tau_{0}, \infty\right)$. Also, following the results in the previous section 5 , we define the prospective super and sub-solutions $\bar{w}_{\epsilon}^{+}$and $\bar{w}_{\epsilon}^{-}$in the inner region by setting

$$
\begin{aligned}
& \bar{w}_{\epsilon}^{+}(\xi, \tau):=\frac{1}{1+\epsilon} \bar{w}_{0}\left(\xi+C_{1}(\tau)\right) \\
& \bar{w}_{\epsilon}^{-}(\xi, \tau):=\frac{1}{1-\epsilon} \bar{w}_{0}\left(\xi+C_{2}(\tau)\right) .
\end{aligned}
$$

The small constant $\epsilon \in[0,1)$ will be chosen later. Also, for some fixed $\xi_{1}$ to be determined later, let $C_{1}(\tau), C_{2}(\tau)$ be smooth functions defined on $\tau \geq \tau_{0}$ such that

$$
e^{\gamma \tau} \hat{w}^{ \pm}\left(A+\xi_{1} e^{-\gamma \tau}, \tau\right)=\bar{w}_{\epsilon}^{ \pm}\left(\xi_{1}, \tau\right)
$$

Note that the functions $C_{i}(\tau)$ uniquely exist and are smooth because $\bar{w}_{0}(\cdot)$ is strictly increasing smooth function onto $(0, \infty)$ and $\hat{w}^{ \pm}\left(A+\xi_{1} e^{-\gamma \tau}, \tau\right)$ are positive smooth functions on $\tau \geq \tau_{0}$. Moreover, since $\hat{w}_{2}>0$ and $\theta^{+}>\theta^{-}$, we have $e^{\gamma \tau} \hat{w}^{+}(A+$ $\left.\xi_{1} e^{-\gamma \tau}, \tau\right)>e^{\gamma \tau} \hat{w}^{-}\left(A+\xi_{1} e^{-\gamma \tau}, \tau\right)$. Therefore 6.2 and the definition of $w_{\epsilon}^{ \pm}$imply that $\bar{w}_{0}\left(\xi_{1}+C_{1}(\tau)\right)>\bar{w}_{0}\left(\xi_{1}+C_{2}(\tau)\right)$. Using again that $\bar{w}_{0}(\cdot)$ is a strictly increasing we conclude that

$$
C_{1}(\tau)>C_{2}(\tau), \quad \tau \geq \tau_{0}
$$

which will be used later.

It follows from the above discussion that for $\tau \geq \tau_{0}$, we can glue the functions $e^{\gamma \tau} \hat{w}^{ \pm}\left(A+\xi e^{-\gamma \tau}, \tau\right)$ and $\bar{w}_{\epsilon}^{ \pm}(\xi, \tau)$ at $\xi=\xi_{1}$ to form a continuous and piecewise smooth function, namely we define

$$
\begin{aligned}
& w_{\epsilon}^{+}(\xi, \tau):= \begin{cases}\bar{w}_{\epsilon}^{+}(\xi, \tau) & \text { if } \xi \leq \xi_{1} \\
e^{\gamma \tau} \hat{w}^{+}\left(A+\xi e^{-\gamma \tau}, \tau\right) & \text { if } \xi>\xi_{1}\end{cases} \\
& w_{\epsilon}^{-}(\xi, \tau):= \begin{cases}\bar{w}_{\epsilon}^{-}(\xi, \tau) & \text { if } \xi \leq \xi_{1} \\
e^{\gamma \tau} \hat{w}^{-}\left(A+\xi e^{-\gamma \tau}, \tau\right) & \text { if } \xi>\xi_{1}\end{cases}
\end{aligned}
$$

(see Figure 2 below).

We will show next that the functions $w_{\epsilon}^{+}(\xi, \tau)$ and $w_{\epsilon}^{-}(\xi, \tau)$ have the following properties:

Proposition 6.1. There exist $\xi_{1}>0$ and $\epsilon_{1}>0$ such that for any $0<\epsilon<\epsilon_{1}$ there is a $\tau_{1}=\tau_{1}(\epsilon)$ for which the functions $w_{\epsilon}^{+}$and $w_{\epsilon}^{-}$given by (6.4) with $0<\epsilon<\epsilon_{1}$, have following properties:

(i) $w_{\epsilon}^{+}(\xi, \tau)>w_{\epsilon}^{-}(\xi, \tau)>0$ on $(-\infty, \infty) \times\left[\tau_{1}, \infty\right)$;

(ii) $w_{\epsilon}^{+}(\xi, \tau)$ and $w_{\epsilon}^{-}(\xi, \tau)$ are continuous on $(-\infty, \infty) \times\left[\tau_{1}, \infty\right)$ and smooth for $\xi \neq \xi_{1}$

(iii) for all $(\xi, \tau)$ with $\xi \neq \xi_{1}$ and $\tau \geq \tau_{1}$, they satisfy $I\left[w_{\epsilon}^{+}\right]>0$ and $I\left[w_{\epsilon}^{-}\right]<0$, i.e. they are super and sub-solutions, respectively;

(iv) at the non-smooth points $\left(\xi_{1}, \tau\right), \tau \geq \tau_{1}$, they satisfy

$$
\begin{aligned}
& \lim _{\xi \rightarrow \xi_{1}-} \frac{\partial}{\partial \xi} w_{\epsilon}^{+}(\xi, \tau)>\lim _{\xi \rightarrow \xi_{1}+} \frac{\partial}{\partial \xi} w_{\epsilon}^{+}(\xi, \tau) \\
& \lim _{\xi \rightarrow \xi_{1}-} \frac{\partial}{\partial \xi} w_{\epsilon}^{-}(\xi, \tau)<\lim _{\xi \rightarrow \xi_{1}+} \frac{\partial}{\partial \xi} w_{\epsilon}^{-}(\xi, \tau) .
\end{aligned}
$$

For the proof of the proposition we will need the next two lemmas. 


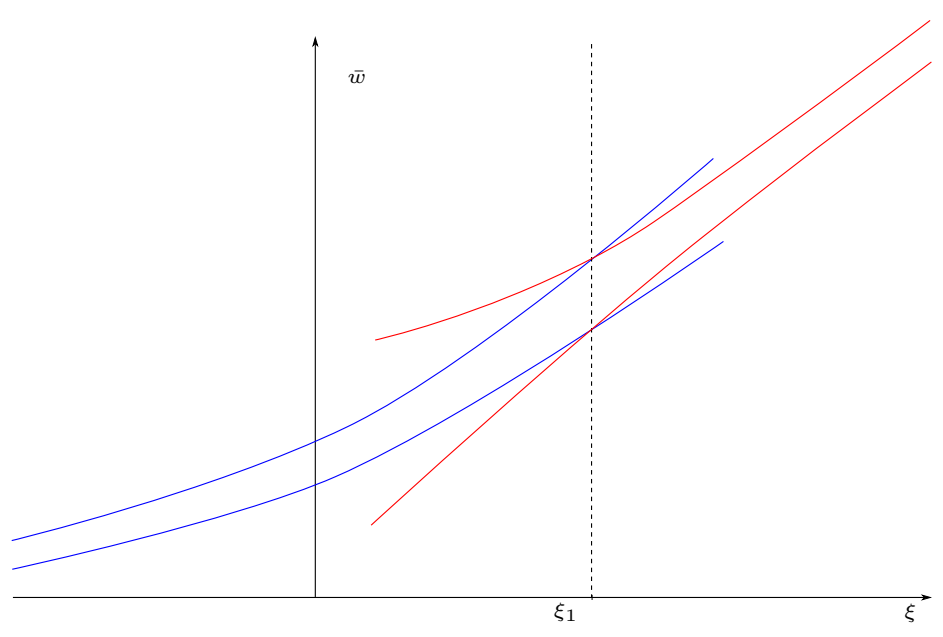

Figure 2

Lemma 6.2. For any fixed $\xi_{1}>\xi_{0}$, we have

$$
e^{\gamma \tau} \hat{w}^{ \pm}\left(A+\xi_{1} e^{-\gamma \tau}, \tau\right) \rightarrow \frac{(n-1)(n-2)}{A \gamma} \xi_{1}+\frac{(n-1) \theta^{ \pm}}{A \gamma} \frac{1}{\xi_{1}}
$$

and

$$
\partial_{\xi}\left[e^{\gamma \tau} \hat{w}^{ \pm}\left(A+\xi e^{-\gamma \tau}, \tau\right)\right]_{\xi=\xi_{1}} \rightarrow \frac{(n-1)(n-2)}{A \gamma}-\frac{(n-1) \theta^{ \pm}}{A \gamma} \frac{1}{\xi_{1}^{2}}
$$

as $\tau \rightarrow \infty$.

Proof. We have that $\lim _{\tau \rightarrow+\infty} e^{\gamma \tau} \hat{w}_{0}\left(A+\xi_{1} e^{-\gamma \tau}\right)=\frac{(n-1)(n-2)}{A \gamma} \xi_{1}$ from Taylor's theorem on $\hat{w}_{0}$ at $\xi=A$ and, for $\gamma>\frac{1}{2}, \lim _{\tau \rightarrow+\infty} e^{-\gamma \tau} \hat{h}\left(A+\xi_{1} e^{-\gamma \tau}\right)=\frac{(n-1) \theta}{A \gamma} \frac{1}{\xi_{1}}$ from Lemma 4.1. This proves the first statement of the lemma for $\gamma>\frac{1}{2}$. Similarly, Taylor's Theorem on $\hat{w}_{0}^{\prime}$ and Lemma 4.1 imply the second statement for $\gamma>\frac{1}{2}$. In the case where $0<\gamma \leq 1 / 2$ a statement similar to Lemma 4.1 clearly holds for the modified $\hat{h}(\eta, \tau)$ and the result follows in the same way.

Although we haven't chosen $\xi_{1}$ yet, we will next check that $w^{+}$stays above $w^{-}$, for all small $\epsilon>0$.

Lemma 6.3. For any fixed $\xi_{1}$ and $\tau_{0} \in \mathbb{R}$, there exists $\epsilon_{0}=\epsilon_{0}\left(\xi_{1}, \tau_{0}\right)>0$ such that for all $0<\epsilon<\epsilon_{0}$,

$$
w_{\epsilon}^{+}(\xi, \tau)>w_{\epsilon}^{-}(\xi, \tau), \quad \text { for }(\xi, \tau) \in(-\infty, \infty) \times\left[\tau_{0},+\infty\right) .
$$


Proof. Since $\hat{w}_{2}$ is a positive function, we have $\hat{w}^{+}>\hat{w}^{-}$and hence by the definition 6.1) we have

$$
w_{\epsilon}^{+}>w_{\epsilon}^{-} \quad \text { on } \xi \geq \xi_{1} \text { and } \tau \geq \tau_{0} .
$$

On the other hand, it is obvious from the definition of $\bar{w}_{\epsilon}^{ \pm}$that

$$
\bar{w}_{\epsilon}^{+}>\frac{1}{1+\epsilon} \bar{w}_{0}^{+} \text {and } \frac{1}{1-\epsilon} \bar{w}_{0}^{-}>\bar{w}_{\epsilon}^{-}
$$

in the remaining region $\xi<\xi_{1}$ and $\tau \geq \tau_{0}$. Here, $\bar{w}_{0}^{+}, \bar{w}_{0}^{-}$are $\bar{w}_{\epsilon}^{+}, \bar{w}_{\epsilon}^{-}$with $\epsilon=0$. Thus, it suffices to find small $\epsilon_{0}>0$, depending on $\xi_{1}$, such that

$$
\frac{\bar{w}_{0}^{+}(\xi, \tau)}{1+\epsilon_{0}}>\frac{\bar{w}_{0}^{-}(\xi, \tau)}{1-\epsilon_{0}} \quad \text { on } \xi \leq \xi_{1} \quad \text { and } \quad \tau \geq \tau_{0} .
$$

To this end, we will first show that if $C_{1,0}, C_{2,0}$ are defined by 6.2 when $\epsilon=0$, then $C_{1,0}>C_{2,0}$ and, as $\tau \rightarrow+\infty$,

$$
C_{0,1}(\tau) \rightarrow C_{0,1, \infty}, \quad C_{0,2}(\tau) \rightarrow C_{0,2, \infty} \quad \text { with } \quad C_{0,1, \infty}>C_{0,2, \infty} .
$$

Indeed, this readily follows from the definition $\sqrt{6.2}, \hat{w}^{+}>\hat{w}^{-}$and the fact that as $\tau \rightarrow+\infty$,

$$
e^{\gamma \tau} \hat{w}^{ \pm}\left(A+\xi_{1} e^{-\gamma \tau}, \tau\right) \rightarrow \frac{(n-1)(n-2)}{A \gamma} \xi_{1}+\frac{(n-1) \theta^{ \pm}}{A \gamma} \frac{1}{\xi_{1}},
$$

with $\theta^{+}>\theta^{-}$. This in particular implies $\bar{w}_{0}^{+}>\bar{w}_{0}^{-}$.

To conclude (6.7), we will now use $(6.9)$ and the fact that under the coordinate change 1.5 where $\xi=\ln r$ the functions $\bar{w}_{0}^{ \pm}(\xi, \tau)$ are mapped into the functions $\bar{U}^{+}(r, \tau)>\bar{U}^{-}(r, \tau)$ given by

$$
\begin{aligned}
& \left(\bar{U}^{+}\right)^{1-m}(r, \tau):=r^{-2} \bar{w}_{0}^{+}(\ln r, \tau)=e^{2 C_{0,1}(\tau)} \bar{U}^{1-m}\left(r e^{C_{0,1}(\tau)}\right) \\
& \left(\bar{U}^{-}\right)^{1-m}(r, \tau):=r^{-2} \bar{w}_{0}^{-}(\ln r, \tau)=e^{2 C_{0,2}(\tau)} \bar{U}^{1-m}\left(r e^{C_{0,2}(\tau)}\right)
\end{aligned}
$$

where under this transformation the region $\xi \leq \xi_{1}$ corresponds to the compact region $\left\{x \in \mathbb{R}^{n}|r=| x \mid \leq e^{\xi_{1}}\right\}$ of $\mathbb{R}^{n}$. Here, recall that $\bar{U}^{1-m}(r)=r^{-2} \bar{w}_{0}(\ln r)$. We then conclude, using 6.9 that there exists small $\epsilon_{0}\left(\xi_{1}, \tau_{0}\right)$ such that

$$
\frac{1}{1+\epsilon_{0}}\left(\bar{U}^{+}\right)^{1-m}>\frac{1}{1-\epsilon_{0}}\left(\bar{U}^{-}\right)^{1-m} \quad \text { on } r \leq e^{\xi_{1}} \text { and } \tau \geq \tau_{0}
$$

showing 6.8).

We are now ready to proceed to the proof of Proposition 6.1.

Proof of Proposition 6.1. We have to find $\xi_{1}, \epsilon_{1}$ and $\tau_{1}(\epsilon)$ for each $0<\epsilon<\epsilon_{1}$. Notice that $\xi_{0}$ and $\tau_{0}$ come from Proposition 4.4 and 4.7 and they are fixed throughout the proof. As long as $\tau>\tau_{0}$ and $0<\epsilon<1, w_{\epsilon}^{+}$and $w_{\epsilon}^{-}$are well defined and part (ii) follows from their construction. For $\xi_{1}>\xi_{0}>0$ to be determined later, we have $\epsilon_{0}\left(\xi_{1}\right)>0$ from Lemma 6.3 so that part (i) is true for $0<\epsilon<\epsilon_{0}$ and $\tau>\tau_{0}$. In summary, we may choose $\epsilon_{1} \leq \epsilon_{0}\left(\xi_{1}\right)$ and any $\tau(\epsilon)>\tau_{0}$ for undetermined $\xi_{1}>\xi_{0}>0$ so that part (i) and (ii) are always true. Before going to show (iv), let us recall asymptotic properties of $\bar{w}_{0}$ shown in [CD. As $\xi \rightarrow \infty$, we have

$$
\begin{aligned}
& \bar{w}_{0}(\xi)=\frac{(n-1)(n-2)}{A \gamma} \xi+\frac{(n-1)(n-6)}{4 A \gamma} \frac{1}{\xi}+o\left(\xi^{-1}\right) \\
& \bar{w}_{0}^{\prime}(\xi)=\frac{(n-1)(n-2)}{A \gamma}-\frac{(n-1)(n-6)}{4 A \gamma} \frac{1}{\xi^{2}}+o\left(\xi^{-2}\right) .
\end{aligned}
$$


Let us just check that $\lim _{\xi \rightarrow \xi_{1}-}\left(w_{\epsilon}^{+}\right)_{\xi}(\xi, \tau)>\lim _{\xi \rightarrow \xi_{1}+}\left(w_{\epsilon}^{+}\right)_{\xi}(\xi, \tau)$, as a similar argument holds for the other inequality. By the gluing condition and Lemma 6.2 . we have that for $\xi_{1}>\xi_{0}$,

$$
w_{\epsilon}^{+}\left(\xi_{1}, \tau\right)=(1+\epsilon)^{-1} \bar{w}_{0}\left(\xi_{1}+C_{1}(\tau)\right) \rightarrow \frac{(n-1)(n-2)}{A \gamma} \xi_{1}+\frac{(n-1) \theta^{ \pm}}{A \gamma} \frac{1}{\xi_{1}}
$$

as $\tau \rightarrow \infty$. Let's assume $\epsilon<1$. Invoking 6.10 , we may choose a large $\xi_{1}>\xi_{0}$ so that the following holds independently from $\epsilon$

- $\lim \sup _{\tau \rightarrow \infty}\left|C_{1}(\tau)-\epsilon \xi_{1}\right| \leq 1$

- $\liminf _{\tau \rightarrow \infty}(1+\epsilon)^{-1} \bar{w}_{0}^{\prime}\left(\xi_{1}+C_{1}(\tau)\right)>\frac{(n-1)(n-2)}{(1+\epsilon) A \gamma}-\frac{(n-1)}{(1+\epsilon) A \gamma} \frac{2^{-1}\left(\theta^{+}+\frac{n-6}{4}\right)}{\left((1+\epsilon) \xi_{1}\right)^{2}}$.

Continuing with this choice of $\xi_{1}$, we may find small $\epsilon_{1}>0$ so that $\epsilon_{1}<\min \left(\epsilon_{0}\left(\xi_{1}\right), 1\right)$ and, for all $\epsilon<\epsilon_{1}$,

$$
\frac{(n-1)(n-2)}{(1+\epsilon) A \gamma}-\frac{(n-1)}{(1+\epsilon) A \gamma} \frac{2^{-1}\left(\theta^{+}+\frac{n-6}{4}\right)}{\left((1+\epsilon) \xi_{1}\right)^{2}}>\frac{(n-1)(n-2)}{A \gamma}-\frac{(n-1)}{A \gamma} \frac{\theta^{+}}{\xi_{1}^{2}}
$$

Since

and

$$
\lim _{\xi \rightarrow \xi_{1}-} \frac{\partial}{\partial \xi} w_{\epsilon}^{+}(\xi, \tau)=(1+\epsilon)^{-1} \bar{w}_{0}^{\prime}\left(\xi_{1}+C_{1}(\tau)\right)
$$

$$
\lim _{\xi \rightarrow \xi_{1}+} \frac{\partial}{\partial \xi} w_{\epsilon}^{+}(\xi, \tau)=\partial_{\xi}\left[e^{\gamma \tau} \hat{w}^{+}\left(A+\xi e^{-\gamma \tau}, \tau\right)\right]_{\xi=\xi_{1}},
$$

the second part of Lemma 6.2 and above observation proves (iv) for a large $\tau_{1}$.

In showing (iii), we only need to check this in the inner region as we assume $\xi_{1}>\xi_{0}$ and $\tau_{1}>\tau_{0}$. By Proposition 5.1, it suffices to show for each fixed $\xi_{1}$ and $0<\epsilon<\epsilon_{1}$ there exists $\tau_{1} \gg 1$ such that

$$
\left|C_{1}^{\prime}(\tau)\right| \leq M \quad \text { and } \quad\left|C_{2}^{\prime}(\tau)\right| \leq M, \quad \text { for } \tau \geq \tau_{0}
$$

for some constant $M$. We will actually show that $\lim _{\tau \rightarrow+\infty} C_{i}^{\prime}(\tau)=0, i=1,2$ which yields 6.11 . Lets prove this for $C_{1}$, as the proof for $C_{2}$ is identical. Recall that

$$
0<e^{\gamma \tau} \hat{w}^{+}\left(A+\xi_{1} e^{-\gamma \tau}, \tau\right)=\bar{w}_{\epsilon}^{+}\left(\xi_{1}, \tau\right)=\frac{1}{1+\epsilon} \bar{w}_{0}\left(\xi_{1}+C_{1}(\tau)\right)
$$

For $\gamma>1 / 2$, differentiating in $\tau$ the LHS using that $e^{\gamma \tau} \hat{w}^{+}=e^{\gamma \tau} \hat{w}_{0}+e^{-\gamma \tau} \hat{h}$, we obtain

$$
\mathrm{LHS}=\gamma\left(e^{\gamma \tau} \hat{w}_{0}-\xi_{1} \hat{w}_{0}^{\prime}\right)\left(A+\xi_{1} e^{-\gamma \tau}\right)-\gamma\left(e^{-\gamma \tau} \hat{h}+\xi_{1} e^{-2 \gamma \tau} \hat{h}^{\prime}\right)\left(A+\xi_{1} e^{-\gamma \tau}\right) .
$$

Both terms converge to zero, as $\tau \rightarrow \infty$, by Taylor's theorem for $\hat{w}_{0}, \hat{w}_{0}^{\prime}$ and the asymptotics in Lemma 4.1. The same convergence could be proven similarly for $0<\gamma \leq 1 / 2$ as additional terms multiplied by $e^{\gamma \tau}$ are very small and their $\tau$ derivatives converges to zero at the point $\left(A+\xi_{1} e^{-\gamma \tau}, \tau\right)$. At the same time, for any $\gamma>0$, if we take derivative of RHS we obtain

$$
\mathrm{RHS}=\frac{1}{1+\epsilon} \bar{w}_{0}^{\prime}\left(\xi_{1}+C_{1}(\tau)\right) C_{1}^{\prime}(\tau)
$$

Since the smooth function $C_{1}(\tau)$ converges as $\tau \rightarrow \infty$ and hence

$$
\bar{w}_{0}^{\prime}\left(\xi_{1}+C_{1}(\tau)\right) \rightarrow \bar{w}_{0}^{\prime}\left(\xi_{1}+\lim _{\tau \rightarrow \infty} C_{1}(\tau)\right)>0
$$

this concludes that $C_{1}^{\prime}(\tau) \rightarrow 0$ as $\tau \rightarrow \infty$ and hence bounded for $\tau \gg 1$. The same argument also applies to $C_{2}(\tau)$. Thus 6.11 holds. 
Finally, by the arguments above and Propositions 4.4, 4.7 and 5.1, we can find $\tau_{1} \geq \tau_{0}$ which makes all the statements in our proposition true.

We will finish this section with the following result which is an immediate consequence of the comparison principle and Proposition 6.1.

Theorem 6.4. Let $\xi_{1}, \epsilon_{1}$ and $\tau_{1}=\tau_{1}(\epsilon)$ are such Proposition 6.1 holds. Assume that a given conformally flat initial metric $g_{0}=u_{0}^{1-m}(x) \delta_{i j}$ is bounded above and below by $w_{\epsilon}^{+}(\xi,-\ln T)$ and $w_{\epsilon}^{-}(\xi,-\ln T)$, for some $0<\epsilon<\epsilon_{1}$ and $0<T<e^{-\tau_{1}}$, via the coordinate change

$$
w(\xi, \tau)=|x|^{2} u(x, t), \quad \xi=\ln |x|-A e^{\gamma \tau}, \quad \tau=-\ln (T-t)
$$

at $t=0$. That is

$$
w_{\epsilon}^{-}(\xi,-\ln T) \leq \frac{|x|^{2} u_{0}^{1-m}(x)}{T^{1+\gamma}} \leq w_{\epsilon}^{+}(\xi,-\ln T)
$$

holds, with $\xi=\ln |x|-A T^{-\gamma}$. Then, the solution of the Yamabe flow 1.2 exists on the time interval $(0, T)$ and it is bounded between $w_{\epsilon}^{+}(\xi, \tau)$ and $w_{\epsilon}^{-}(\xi, \tau)$, that is

$$
w_{\epsilon}^{-}(\xi,-\ln (T-t)) \leq \frac{|x|^{2} u^{1-m}(x, t)}{(T-t)^{1+\gamma}} \leq w_{\epsilon}^{+}(\xi,-\ln (T-t))
$$

with $\xi=\ln |x|-A(T-t)^{-\gamma}$

Proof. Immediate by Proposition 6.1 and the comparison principle.

\section{Asymptotic SHAPE OF THE SINGULARITY IN THE INNER REGION AND GEOMETRIC PROPERTIES}

Throughout this section we will fix $\xi_{1}>0$ and $\epsilon>0$ so that $w_{\epsilon}^{+}(\xi, \tau)$ and $w_{\epsilon}^{-}(\xi, \tau)$ given by (6.4) are barriers in view of Proposition 6.1 and Theorem 6.4. To simplify the notation we will denote them by $w^{+}(\xi, \tau)$ and $w^{-}(\xi, \tau)$ respectively. They are super and sub-solutions of equation $(2.3)$ on $\mathbb{R} \times\left[\tau_{1}, \infty\right)$, respectively.

We will first prove that if our initial conformally flat metric of the Yamabe flow (1.2) $u_{0}(\cdot)$ is bounded from above and below by $w^{+}(\cdot,-\ln T)$ and $w^{-}(\cdot,-\ln T)$, for some $-\ln T \geq \tau_{1}$ (c.f. 6.13), then the rescaled solution converges to a steady gradient soliton $\bar{w}_{0}(\xi)$, which is the unique entire solution of the equation 3.8 with asymptotic behavior 3.9 as $\xi \rightarrow \infty$.

Since we are not assuming that our solution $u(x, t)$ of 1.2 is radially symmetric, it is more convenient to work in euclidean coordinates on $\mathbb{R}^{n}$, rather than cylindrical coordinates. We have seen that in order to see the steady state $\bar{w}_{0}$ in the inner region one needs to perform the coordinate change 2.11 on radially symmetric solutions in cylindrical coordinates. Under the transformation 1.5 which brings us back to the plane, this change of variables corresponds the coordinate change (3.1)-(3.2) which transforms a solution $u(x, t)$ of 1.2 to a solution $\bar{u}(y, l)$ of equation

$$
\partial_{l} \bar{u}-\frac{1+\gamma}{(1-m) \gamma} \frac{\bar{u}}{l}=\frac{n-1}{m} \Delta_{y} \bar{u}^{m}+\gamma A\left(y \cdot \nabla_{y} \bar{u}\right)+\frac{2 \gamma A}{1-m} \bar{u} .
$$

We denote $\bar{U}(y)$ the steady soliton $\bar{w}_{0}$ in euclidean coordinates, namely

$$
\bar{U}(y)^{1-m}=|y|^{-2} \bar{w}_{0}(\ln |y|) .
$$


This is the unique radial solution of

$$
\frac{n-1}{m} \Delta u^{m}+\gamma A(y \cdot \nabla u)+\frac{2 \gamma A}{1-m} u=0
$$

with asymptotic behavior

$$
u^{1-m}(y)=\frac{1}{|y|^{2}}\left(\frac{(n-1)(n-2)}{\gamma A} \ln |y|+o(1)\right) .
$$

We will next prove the following result.

Theorem 7.1. Under the assumptions of Theorem 6.4 the rescaled solution $\bar{u}(y, l)$ converges, as $l \rightarrow+\infty$, smoothly on compact sets of $\mathbb{R}^{n}$ to the radial steady soliton $\bar{U}^{1-m}(y)$.

Proof. Let $l_{0}:=\gamma^{-1} T^{-\gamma}$ be the initial rescaled time, corresponding to $t=0$. By Theorem 6.4, for $l>l_{0}>0$ we have

$$
|y|^{-2} w^{-}(\ln |y|, \tau) \leq \bar{u}^{1-m}(y, l) \leq|y|^{-2} w^{+}(\ln |y|, \tau), l=\frac{e^{\gamma \tau}}{\gamma} .
$$

These two bounds give upper and lower bounds away from zero for $\bar{u}(\cdot, l)$ on every compact set in $\mathbb{R}^{n}$ which are uniform in time $l \geq l_{0} \gg 1$. Hence, by standard higher order regularity estimates for uniformly parabolic equations and a compactness argument, we conclude that for any sequence $l_{i} \rightarrow \infty$, the solutions $\bar{u}_{i}(y, l):=$ $\bar{u}\left(y, l_{i}+l\right)$ converge, passing to a subsequence, to a limit $\bar{u}_{\infty}(y, l)$. The convergence is smooth on compact subsets of $\mathbb{R}^{n} \times \mathbb{R}$. Therefore, in view of (7.1) and the uniform local upper bound of our sequence, the limit $\bar{u}_{\infty}$ is a smooth eternal solution of

$$
\partial_{l} \bar{u}=\frac{n-1}{m} \Delta_{y} \bar{u}^{m}+\gamma A\left(y \cdot \nabla_{y} \bar{u}\right)+\frac{2 \gamma A}{1-m} \bar{u} .
$$

To finish the proof we need to show that

$$
\bar{u}_{\infty}(y, l)=\bar{U}(y)
$$

which would also imply that our limit is unique, thus concluding that $\bar{u}(\cdot, l) \rightarrow \bar{U}$, as $l \rightarrow \infty$. To this end, we first observe that by our barrier construction (6.4), we have

$$
w^{ \pm}(\ln |y|, \tau) \rightarrow \frac{(n-1)(n-2)}{\gamma A} \ln |y|+\frac{(n-1)}{A \gamma} \theta^{ \pm} \frac{1}{\ln |y|}
$$

as $\tau \rightarrow+\infty$, uniformly on $e^{\xi_{1}} \leq|y| \leq K$, for any fixed $K>e^{\xi_{1}}$. In particular, this implies that our limit $\bar{u}_{\infty}^{1-m}$ has these bounds and thus

$$
\bar{u}_{\infty}^{1-m}(\cdot, l)=\frac{1}{|y|^{2}}\left(\frac{(n-1)(n-2)}{\gamma A} \ln |y|+o(1)\right)
$$

as $|y| \rightarrow \infty$ uniformly in $l \in \mathbb{R}$. For $\lambda>0$, if we denote $\bar{U}_{\lambda}(y):=\lambda^{\frac{2}{1-m}} \bar{U}(\lambda y)$, this is again a radial solution of 7.2 with

$$
\bar{U}_{\lambda}^{1-m}(y)=\frac{1}{|y|^{2}}\left(\frac{(n-1)(n-2)}{\gamma A} \ln |y|+\ln \lambda+o(1)\right) .
$$

This is just a time translation of the radial steady soliton $\bar{U}$ and they are isometric. Since on the soliton the scalar curvature $R>0$ everywhere, the solution pointwise 
decreases as time increases and hence $\bar{U}_{\lambda_{1}}>\bar{U}_{\lambda_{2}}$ for $\lambda_{1}>\lambda_{2}$. Thus we may define

$$
\begin{aligned}
& \lambda_{+}:=\inf \left\{\lambda>0 \mid \bar{U}_{\lambda}(\cdot) \geq \bar{u}_{\infty}(\cdot, l) \text { for all } l\right\} \\
& \lambda_{-}:=\sup \left\{\lambda>0 \mid \bar{U}_{\lambda}(\cdot) \leq \bar{u}_{\infty}(\cdot, l) \text { for all } l\right\} .
\end{aligned}
$$

Our proof will finish if we show that $\lambda_{+}=\lambda_{-}=1$. Let us prove that $\lambda_{+}=1$. Since $\inf _{B\left(e^{\left.\xi_{1}, 0\right)}\right.} \bar{U}_{\lambda} \rightarrow \infty$ as $\lambda \rightarrow \infty$ (see the observation in Corollary 3.3 in [CD]), the construction of $w^{+}$in the inner region and (7.7) imply that we can find large $\lambda>1$ such that $\bar{U}_{\lambda}(\cdot)>\bar{u}_{\infty}(\cdot, l)$ for all $l$. By $(7.6)$ and $(7.7), \bar{U}_{\lambda}(\cdot) \nsupseteq \bar{u}_{\infty}(\cdot, l)$ for $\lambda<1$. Therefore, $\lambda_{+}$is a well defined number with $\lambda_{+} \geq 1$. Assume that $\lambda_{+}>1$. For each $\bar{U}_{\lambda_{+}-2^{-n}}$, there is a point $\left(x_{n}, l_{n}\right)$ with $\bar{U}_{\lambda_{+}-2^{-n}}\left(x_{n}\right)<\bar{U}_{\infty}\left(x_{n}, l_{n}\right)$. Moreover, the sequence of points $\left\{x_{n}\right\}$ such that $\lambda_{+}-2^{-n}>1$ is bounded due to (7.6) and 7.7). By standard regularity estimates on the equation 7.5 , we can find a subsequence of $\left(x_{n}, l_{n}\right)$ such that

$$
\bar{u}_{n_{j}}^{*}(x, l):=\bar{u}_{\infty}\left(x, l_{n_{j}}+l\right) \rightarrow \bar{u}_{\infty}^{*}(x, l)
$$

smoothly on compact sets and $x_{n_{j}} \rightarrow x^{*}$.

Note that $\bar{U}_{\lambda_{+}}(x) \geq \bar{u}_{\infty}^{*}(x, l)$ for all $l$. On the other hand we have $\bar{U}_{\lambda_{+}}\left(x^{*}\right)=$ $\bar{u}_{\infty}\left(x^{*}, 0\right)$. Hence, by the strong maximum principle, we must have $\bar{U}_{\lambda_{+}}(\cdot)=$ $\bar{u}_{\infty}^{*}(\cdot, l)$, for all $l$. But this can't happen since $7.6,27.7$ holds and we have assumed that $\lambda_{+}>1$. By contradiction, this proves that $\lambda_{+}=1$ and $\lambda_{-}=1 \mathrm{can}$ be shown similarly. This concludes the proof of our theorem.

Let us remark the following.

Remark 7.1 (Scalar curvature blow up rate near the origin). $u(x, t)$ of 1.2 represents conformally flat solution of the Yamabe flow $g(t)=u^{1-m}(x, t) \delta_{i j}$ on $\mathbb{R}^{n}$. The metric of rescaled solution $\bar{u}(y, l)$ can be written as

$$
\bar{u}^{1-m} \delta_{i j}=(T-t)^{-(1+\gamma)} \phi_{t}^{*} g(t)
$$

where $\phi_{t}$ is a one parameter family of diffeomorphisms $\phi_{t}(x)=e^{A(T-t)^{-\gamma}} x$. Therefore, Theorem 7.1 can be rephrased as convergence of the pointed manifold

$$
\left(\mathbb{R}^{n}, \frac{g(t)}{(T-t)^{1+\gamma}}, 0\right) \rightarrow\left(\mathbb{R}^{n}, \bar{U}^{1-m} \delta_{i j}, 0\right)
$$

in Cheeger-Gromov sense. This, in particular, implies that

$$
\left|\operatorname{Rm}_{g(t) /(T-t)^{(1+\gamma)}}\right|\left(e^{A(T-t)^{-\gamma}} y\right) \rightarrow\left|\operatorname{Rm}_{\bar{U}^{1-m}} \delta_{i j}\right|(y)
$$

concluding that

$$
(T-t)^{1+\gamma}\left|\mathrm{Rm}_{g(t)}\right|\left(e^{A(T-t)^{-\gamma}} y\right) \rightarrow\left|\mathrm{Rm}_{\bar{U}^{1-m}} \delta_{i j}\right|(y)
$$

and also

$$
(T-t)^{1+\gamma} R_{g(t)}\left(e^{A(T-t)^{-\gamma}} y\right) \rightarrow R_{\bar{U}^{1-m} \delta_{i j}}(y)
$$

In particular the above implies the following blow up rate of the Riemmannian and Scalar curvature at the origin

$$
\lim _{t \rightarrow T-}(T-t)^{1+\gamma}\left|\operatorname{Rm}_{g(t)}\right|(0)=\left|\mathrm{R} m_{\bar{U}^{1-m} \delta_{i j}}\right|(0)=\frac{2 \gamma A}{\sqrt{n(n-1)}}
$$

and

$$
\lim _{t \rightarrow T-}(T-t)^{1+\gamma} R_{g(t)}(0)=R_{\bar{U}^{1-m} \delta_{i j}}(0)=2 \gamma A
$$


We will next show that the global supremum of the curvature occurs asymptotically at the origin as $t \rightarrow T-$.

Proposition 7.2. Under the assumptions of Theorem 6.4 we have

$$
\lim _{t \rightarrow T-}\left[(T-t)^{1+\gamma} \sup _{x \in \mathbb{R}^{n}}\left|\operatorname{Rm}_{g(t)}\right|\right]=\frac{2 \gamma A}{\sqrt{n(n-1)}}
$$

and if $\left\{\left(x_{i}, t_{i}\right)\right\}$ are points such that $t_{i} \rightarrow T$ and

$$
\lim _{i \rightarrow \infty}\left[\left(T-t_{i}\right)^{1+\gamma}\left|\operatorname{Rm}_{g\left(t_{i}\right)}\right|\left(x_{i}\right)\right]=\frac{2 \gamma A}{\sqrt{n(n-1)}}
$$

then

$$
y_{i}:=e^{-A\left(T-t_{i}\right)^{-\gamma}} x_{i} \rightarrow 0, \quad \text { as } i \rightarrow \infty .
$$

In other words, $(T-t)^{-\frac{1+\gamma}{2}} \operatorname{dist}_{g\left(t_{i}\right)}\left(x_{i}, 0\right) \rightarrow 0$ due to the convergence of the metric.

The result of this proposition follows from the following curvature estimate lemma in the outer region. This lemma is useful in the sense that it also gives the curvature blow up rate in other regions. For example, it shows that the curvature blows up in a type I manner near the infinite cylindrical region.

Lemma 7.3. There exist $C>0, r_{0} \gg 1$ and $0<t_{0}<T$ such that the following holds

$$
(T-t) \hat{w}_{0}\left(A+(T-t)^{\gamma} \ln |y|\right)\left|\operatorname{Rm}_{g(t)}\left(e^{A(T-t)^{-\gamma}} y\right)\right| \leq C
$$

on $|y| \geq r_{0}$ and $t_{0}<t<T$.

Let us first show that Lemma 7.3 implies the proposition and then finish this section by proving the lemma.

Proof of Proposition 7.2. Assuming that the Lemma 7.3 holds, then since $\hat{w}_{0}$ is increasing function, for each fixed $r_{1} \geq r_{0}$ we have

$$
(T-t)^{1+\gamma} \sup _{|y| \geq r_{1}}\left|\operatorname{Rm}_{g(t)}\left(e^{A(T-t)^{-\gamma}} y\right)\right|<\frac{C(T-t)^{\gamma}}{\hat{w}_{0}\left(A+(T-t)^{\gamma} \ln \left|r_{1}\right|\right)}
$$

and, by the Taylor expansion of $\hat{w}_{0}(\cdot)$ at $A$, taking the limit $t \rightarrow T-$, yields

$$
\limsup _{t \rightarrow T-}\left[(T-t)^{1+\gamma} \sup _{|y| \geq r_{1}}\left|\operatorname{Rm}_{g(t)}\left(e^{A(T-t)^{-\gamma}} y\right)\right|\right] \leq \frac{C \gamma A}{(n-1)(n-2) \ln r_{1}} .
$$

Choose $r_{1} \geq r_{0}$ sufficiently large so that

$$
\frac{C \gamma A}{(n-1)(n-2) \ln r_{1}}<\frac{2 \gamma A}{\sqrt{n(n-1)}} .
$$

Now on the remaining region $|x| \leq r_{1}$, due to the smooth convergence of $\bar{u}^{1-m}$ to $\bar{U}^{1-m}$ on compact sets and the fact that the steady soliton $\bar{U}^{1-m}$ attains its maximum curvature at the origin we have

$$
\lim _{t \rightarrow T-}\left[(T-t)^{1+\gamma} \sup _{|y| \leq r_{1}}\left|R m_{g(t)}\left(e^{A(T-t)^{-\gamma}} y\right)\right|\right]=\frac{2 \gamma A}{\sqrt{n(n-1)}}
$$

and also the second statement of the proposition holds, concluding the proof. 
Proof of Lemma 7.3. For $r_{0}$ and $t_{0}$ to be chosen later, we pick and fix a point $\left(y_{1}, t_{1}\right)$ with $\left|y_{1}\right| \geq r_{0}$ and $t_{0}<t_{1}<T$ and consider the following scaling of the solution

$$
v^{1-m}(z, \sigma):=\left(e^{A\left(T-t_{1}\right)^{-\gamma}}\left|y_{1}\right|\right)^{2} \frac{u^{1-m}\left(e^{A\left(T-t_{1}\right)^{-\gamma}}\left|y_{1}\right| z, t\right)}{(T-t) \hat{w}_{0}\left(A+\left(T-t_{1}\right)^{\gamma} \ln \left|y_{1}\right|\right)}
$$

with

$$
\sigma(t):=\ln \left(\frac{T-t_{1}}{T-t}\right) \frac{1}{\hat{w}_{0}\left(A+\left(T-t_{1}\right)^{\gamma} \ln \left|y_{1}\right|\right)} .
$$

Since $u$ satisfies (1.2), it follows that $v(z, \sigma)$ evolves by

$$
\frac{\partial}{\partial \sigma} v=\frac{n-1}{m} \Delta_{z} v^{m}-\hat{w}_{0}\left(A+\left(T-t_{1}\right)^{\gamma} \ln \left|y_{1}\right|\right) v .
$$

Claim 7.4. There are $r_{0}>1$ and $t_{0}>0$ so that if $\left|y_{1}\right| \geq r_{0}$ and $t_{1} \geq t_{0}$, then there exist positive constants $c$ and $C$ which are independent of $\left(y_{1}, t_{1}\right)$ such that

$$
c \leq v^{1-m}(z, \sigma) \leq C, \quad \text { for }(z, \sigma) \in \mathcal{A} \times[-1,0]
$$

where $\mathcal{A}$ is the annulus $\mathcal{A}=\left\{z \in \mathbb{R}^{n}|1 / 2 \leq| z \mid \leq 3 / 2\right\}$.

Lets us assume that the claim holds and finish the proof of the lemma. Since $\left|\hat{w}_{0}\right| \leq(n-1)(n-2)$, the equation $(7.13)$ is uniformly parabolic on $\mathcal{A} \times[-1,0]$, independently from choice of the point $\left(y_{1}, t_{1}\right)$, and therefore by standard parabolic regularity estimates, we have uniform bounds for $|\nabla v|$ and $\left|\nabla^{2} v\right|$ on any strictly smaller parabolic cylinder. In particular, we have

$$
\left|\nabla v\left(y_{1} /\left|y_{1}\right|, 0\right)\right| \text { and }\left|\nabla^{2} v\left(y_{1} /\left|y_{1}\right|, 0\right)\right|<C .
$$

Since $v^{1-m}(\cdot, 0) \delta_{i j}$ and $u^{1-m}\left(\cdot, t_{1}\right) \delta_{i j}$ are isometric, we conclude that

$$
\begin{aligned}
\left(T-t_{1}\right) \hat{w}_{0}\left(A+\left(T-t_{1}\right)^{\gamma} \ln \left|y_{1}\right|\right) \mid \operatorname{Rm}_{g\left(t_{1}\right)} & \left(e^{A\left(T-t_{1}\right)^{-\gamma}} y_{1}\right) \mid \\
& =\left|\operatorname{Rm}_{v^{1-m}}(0) \delta_{i j}\left(\frac{y_{1}}{\left|y_{1}\right|}\right)\right| \leq C .
\end{aligned}
$$

The constant $C$ is independent from $\left(y_{1}, t_{1}\right)$. This finishes the proof of the lemma.

Proof of the Claim 7.4. Although the computations below might look intimidating, the idea is simple. Since $u$ is trapped between the two barriers in the outer region where $|x| \geq e^{\frac{A}{(T-t) \gamma}} e^{\xi_{1}}$, namely

$$
\hat{w}^{-}\left((T-t)^{\gamma} \ln |x|,-\ln (T-t)\right) \leq \frac{|x|^{2} u(x, t)}{T-t} \leq \hat{w}^{+}\left((T-t)^{\gamma} \ln |x|,-\ln (T-t)\right)
$$

and since $\hat{w}^{+}$and $\hat{w}^{-}$are close to $\hat{w}_{0}$, we expect that different values of $v$ are similar in the whole annulus.

Indeed, suppose $r_{0}>1$ and $t_{0} \in(0, T)$ are first chosen to satisfy

$$
\ln \frac{r_{0}}{2}>\xi_{1} \quad \text { and } \quad-\ln \left(T-t_{0}\right)>-\ln T+(n-1)(n-2) .
$$

With this choice of $t_{0}$ and $0 \leq \hat{w}_{0} \leq(n-1)(n-2)$, we see for that

$$
\sigma(0)=\frac{\ln \left(T-t_{1}\right)-\ln (T-0)}{w_{0}\left(A+\left(T-t_{1}\right)^{\gamma} \ln \left|y_{1}\right|\right)} \leq \frac{\ln \left(T-t_{0}\right)-\ln T}{(n-1)(n-2)} \leq-\frac{(n-1)(n-2)}{(n-1)(n-2)}=-1
$$


where $\sigma(t)$ is defined by 7.12 . Since $u(x, t)$ is defined for $t \geq 0$, this shows that the rescaled function $v(z, \sigma)$ is well defined on $\mathcal{A} \times[-1,0]$. By choosing $r_{0}>2 e^{\xi_{1}}$ sufficiently large and $t_{0} \in(0, T)$ closer to $T$, we may assume that

$$
\frac{1}{2} \hat{w}_{0}\left(A+\xi e^{-\gamma \tau}\right) \leq \hat{w}^{-}\left(A+\xi e^{-\gamma \tau}, \tau\right)
$$

and

$$
\hat{w}^{+}\left(A+\xi e^{-\gamma \tau}, \tau\right) \leq 2 \hat{w}_{0}\left(A+\xi e^{-\gamma \tau}\right)
$$

on $\xi \geq \ln \left(r_{0} / 2\right)$ and $\sigma \geq-1$. This is possible because $\hat{w}^{ \pm}=\hat{w}_{0}+e^{-2 \gamma t} \hat{h}$, where $\hat{h}$ is bounded away from $A$ and satisfies 4.1 near $A$, when $\gamma>1 / 2$. The other range $\gamma \in(0,1 / 2]$ is similar (see Claim 4.9). Using (7.14) we can then estimate

$$
\begin{aligned}
v^{1-m}(z, \sigma) & =\frac{\left(e^{\frac{A}{\left(T-t_{1}\right)^{\gamma}}}\left|y_{1}\right|\right)^{2}}{T-t} \frac{u^{1-m}\left(e^{\frac{A}{(T-t)^{\gamma}}}\left|y_{1}\right| e^{\left.\frac{A}{\left(T-t_{1}\right)^{\gamma}}-\frac{A}{(T-t) \gamma} z, t\right)}\right.}{\hat{w}_{0}\left(A+\left(T-t_{1}\right)^{\gamma} \ln \left|y_{1}\right|\right)} \\
& \leq \frac{1}{|z|^{2}} \frac{\hat{w}^{+}\left(A+(T-t)^{\gamma} \ln \left(\left|y_{1}\right||z| e^{\left.\left.\frac{A}{\left(T-t_{1}\right)^{\gamma}}-\frac{A}{(T-t)^{\gamma}}\right), \tau\right)}\right.\right.}{\hat{w}_{0}\left(A+\left(T-t_{1}\right)^{\gamma} \ln \left|y_{1}\right|\right)} \\
& \leq \frac{2}{|z|^{2}} \frac{\hat{w}_{0}\left(A+(T-t)^{\gamma}\left(\ln \left|y_{1}\right|+\ln |z|\right)+A\left(\frac{(T-t)^{\gamma}}{\left(T-t_{1}\right)^{\gamma}}-1\right)\right)}{\hat{w}_{0}\left(A+\left(T-t_{1}\right)^{\gamma} \ln \left|y_{1}\right|\right)} .
\end{aligned}
$$

Since $t \leq t_{1}$ and $\left|y_{1}\right| \geq r_{0}$, we have $\left|y_{1}\right||z| e^{\frac{A}{\left(T-t_{1}\right)^{\gamma}}-\frac{A}{(T-t)^{\gamma}}} \geq r_{0} \cdot(1 / 2) \cdot 1 \geq e^{\xi_{1}}$, thus we could bound above $v^{1-m}(z, \sigma)$ using our barrier $\hat{w}^{+}$of the outer region in the second line and use $(7.16)$ in the last line. Similarly we get a lower bound

$$
v^{1-m}(z, \sigma) \geq \frac{1}{2|z|^{2}} \frac{\hat{w}_{0}\left(A+(T-t)^{\gamma}\left(\ln \left|y_{1}\right|+\ln |z|\right)+A\left(\frac{(T-t)^{\gamma}}{\left(T-t_{1}\right)^{\gamma}}-1\right)\right)}{\hat{w}_{0}\left(A+\left(T-t_{1}\right)^{\gamma} \ln \left|y_{1}\right|\right)} .
$$

If we could find constants $0<c<1<C$ such that

$$
c \leq \frac{(T-t)^{\gamma}\left(\ln \left|y_{1}\right|+\ln |z|\right)+A\left(\frac{(T-t)^{\gamma}}{\left(T-t_{1}\right)^{\gamma}}-1\right)}{(T-t)^{\gamma} \ln \left|y_{1}\right|} \leq C
$$

holds on $(v, \sigma) \in A \times[-1,0]$, then since $\hat{w}_{0}(A+x)$ is an increasing function on $x \geq 0$, concave and $\hat{w}_{0}(A+0)=0$, we would deduce that

$$
c \leq \frac{\hat{w}_{0}\left(A+(T-t)^{\gamma}\left(\ln \left|y_{1}\right|+\ln |z|\right)+A\left(\frac{(T-t)^{\gamma}}{\left(T-t_{1}\right)^{\gamma}}-1\right)\right)}{\hat{w}_{0}\left(A+(T-t)^{\gamma} \ln \left|y_{1}\right|\right)} \leq C
$$

which combined with the above would finish the proof of our claim.

Now let us now find the bounds (7.17). First, it is clear that

$$
1+\frac{\ln \frac{1}{2}}{\ln r_{0}} \leq \frac{\ln \left|y_{1}\right|+\ln |z|}{\ln \left|y_{1}\right|} \leq 1+\frac{\ln \frac{3}{2}}{\ln r_{0}}
$$

and notice that $1+\frac{\ln \frac{1}{2}}{\ln r_{0}}=\frac{\ln \frac{r_{0}}{2}}{\ln r_{0}}$ is positive since $\ln \frac{r_{0}}{2}>\xi_{1}>0$. Thus, it suffices to prove

$$
0<\frac{A\left(\frac{(T-t)^{\gamma}}{\left(T-t_{1}\right)^{\gamma}}-1\right)}{(T-t)^{\gamma} \ln \left|y_{1}\right|}<C_{1}
$$


by some constant $C_{1}<\infty$. If we introduce $\theta:=\left(T-t_{1}\right)^{\gamma} \ln \left|y_{1}\right|>0$, then by the definition of $\sigma$ in 7.12

$$
\frac{(T-t)^{\gamma}}{\left(T-t_{1}\right)^{\gamma}}=e^{-\sigma \gamma \hat{w}_{0}\left(A+\ln \left|y_{1}\right|\left(T-t_{1}\right)^{\gamma}\right)}=e^{-\sigma \gamma \hat{w}_{0}(A+\theta)}>0 .
$$

Now $0 \leq-\sigma \leq 1$ and $e^{-\sigma \gamma \hat{w}_{0}(A+\theta)} \geq 1$ imply

$$
0<\frac{A\left(\frac{(T-t)^{\gamma}}{\left(T-t_{1}\right)^{\gamma}}-1\right)}{(T-t)^{\gamma} \ln \left|y_{1}\right|}=A \frac{e^{-\sigma \gamma \hat{w}_{0}(A+\theta)}-1}{\theta e^{-\sigma \gamma \hat{w}_{0}(A+\theta)}} \leq A \frac{e^{\gamma \hat{w}_{0}(A+\theta)}-e^{\gamma \hat{w}_{0}(A+0)}}{\theta-0} .
$$

By the mean value theorem, there exists $0<\theta_{0}<\theta$ such that

$$
\frac{e^{\gamma \hat{w}_{0}(A+\theta)}-e^{\gamma \hat{w}_{0}(A+0)}}{\theta-0}=\gamma \hat{w}_{0}^{\prime}\left(A+\theta_{0}\right) e^{\gamma \hat{w}_{0}\left(A+\theta_{0}\right)} \leq \gamma \frac{(n-1)(n-2)}{\gamma A} e^{\gamma(n-1)(n-2)} .
$$

Here, we used the facts that $\hat{w}_{0}(A+x)$ is a nonnegative concave function on the set $x \geq 0$ with

$$
\hat{w}_{0}^{\prime}(A)=\frac{(n-1)(n-2)}{\gamma A} \quad \text { and } \quad \hat{w}_{0}(A+x) \leq(n-1)(n-2) .
$$

Combining the last two inequalities implies that 7.19 holds with

$$
C_{1}:=(n-1)(n-2) e^{\gamma(n-1)(n-2)} .
$$

This finished the proof of the claim and also the proof of the lemma.

\section{Proof of Theorem 1.2}

In this final section, we will give the proof of Theorem 1.2 as stated in the introduction. We will need the following rigidity result for eternal solutions of conformally flat Yamabe flow.

Proposition 8.1. Let $g(t)=u^{1-m}(x, t) \delta_{i j}$ be a smooth eternal solution to the conformally flat Yamabe flow (1.2) on $\mathbb{R}^{n} \times(-\infty, \infty)$, with positive Ricci and uniformly bounded sectional curvature. We further assume that $u$ is bounded from below by a radial steady gradient soliton centered at the origin with maximum scalar curvature $2 \gamma A>0$, that is

$$
u(x, t) \geq e^{-\frac{2 \gamma A\left(t+\xi_{1}\right)}{1-m}} \bar{U}\left(|x| e^{-\gamma A\left(t+\xi_{1}\right)}\right) \quad \text { for some } \xi_{1} \in \mathbb{R} .
$$

Then, $u(x, t)$ must be a radial gradient steady soliton, that is

$$
u(x, t) \equiv e^{-\frac{2 \gamma A\left(t+\xi_{0}\right)}{1-m}} \bar{U}\left(|x| e^{-\gamma A\left(t+\xi_{0}\right)}\right) \quad \text { for some } \xi_{0} \leq \xi_{1} .
$$

Proof. By the Harnack inequality for the Yamabe flow (c.f. Theorem 3.7 in [Ch]), for any 1 -form $X_{i}$

$$
(n-1) \Delta R+\langle\nabla R, X\rangle+\frac{1}{n-1} R_{i j} X^{i} X^{j}+R^{2} \geq 0 .
$$

Note that since our solution exists from $t=-\infty$ we could drop $R / t$ term from the original Harnack expression in $\mathrm{Ch}$. This inequality, in particular implies that

$$
(n-1) \Delta R+R^{2}=\partial_{t} R>0 .
$$

Claim 8.2. $R_{g}(0,0)=2 \gamma A$ and $2 \gamma A=\sup R_{g}(x, t)$. 
Proof of Claim 8.2. The proof is simple and uses that $\partial_{t} R>0$ and

$$
\partial_{t} u^{1-m}(x, t)=-R_{g}(x, t) u^{1-m}(x, t) .
$$

Suppose there is a point $\left(x_{0}, t_{0}\right)$ with $R_{g}\left(x_{0}, t_{0}\right)>2 \gamma A$. Since $\partial_{t} R>0$, a ODE comparison implies

$$
u\left(x_{0}, t\right)=C\left(x_{0}\right) e^{-\frac{1}{1-m} R_{g}\left(x_{0}, t_{0}\right) t}, \quad \text { as } t \rightarrow \infty .
$$

On the other hand, this contradicts to

$$
u\left(x_{0}, t\right) \geq \bar{e}^{-\frac{2 \gamma A\left(t+\xi_{1}\right)}{1-m}} \bar{u}_{0}\left(\left|x_{0}\right| e^{-\gamma A\left(t+\xi_{1}\right)}\right) \geq e^{-\frac{2 \gamma A\left(t+\xi_{1}\right)}{1-m}} \inf _{|y| \leq\left|x_{0}\right| e^{-\gamma A\left(t_{0}+\xi_{1}\right)}} \bar{u}_{0}(y)
$$

which holds for $t \geq t_{0}$. Similarly as before, if $R_{g}(0,0)<2 \gamma A$, then

$$
u(0, t)=C_{0} e^{-\frac{1}{1-m} R_{g}(0,0) t}, \quad \text { as } t \rightarrow-\infty
$$

which again contradicts to 8.1 .

According to the classification of conformally flat radial solitons (c.f. Propositions 1.4 and 1.5 in DS2] the one parameter family of solutions

$$
\bar{U}_{\xi}(x, t):=e^{-\frac{2 \gamma A(t+\xi)}{1-m}} \bar{U}\left(|x| e^{-\gamma A(t+\xi)}\right), \quad \xi \in \mathbb{R}
$$

are all possible conformally flat radial steady gradient solitons whose maximum scalar curvature is $2 \gamma A$ at the origin. It also is known that these solutions attain a strict curvature maximum at the origin. Meanwhile, due to the Claim $8.2 g(x, t)$ attains its maximum scalar curvature at an interior space-time point $(0,0)$. Furthermore, since $g(x, t)$ has positive Ricci curvature and bounded sectional curvature, Corollary 5.1 in DS2 implies that $g(x, t)$ must be a steady gradient soliton. Also, under the nonnegative Ricci condition, such steady gradient solitons are (globally) conformally flat and radially symmetric (c.f. Theorem 3.2 and Corollary 3.3 in CMM] or Corollary 1.6 and Remark 1.2 in [CSZ]). Since $g(x, t)$ has its maximum scalar curvature at the origin, it must be symmetric with respect to this point. In view of Liouville's rigidity theorem on conformal mappings on $\mathbb{R}^{n}$ with $n \geq 3$, $u(x, t)$ must be a radially symmetric function which represents a steady gradient soliton. Hence $u(x, t)=\bar{U}_{\xi_{0}}(x, t)$ by the classification theorem in DS2].

We are now in position to finally give the proof of our main result, Theorem 1.2 ,

Proof of Theorem 1.2. We begin by fixing $\xi_{1}, \epsilon \leq \epsilon_{1}$ and $\tau_{1}$, as they appear in Proposition 6.1 and Theorem 6.4 Set $T_{1}:=e^{-\tau_{1}}$ and fix $T$ with $0<T<T_{1}$.

Claim 8.3. There exist $\xi_{a}>\xi_{b}$ so that

$$
w_{\epsilon}^{+}\left(\xi-\gamma A \xi_{a},-\ln T\right) \leq \frac{|x|^{2} u_{0}^{1-m}(x)}{T^{1+\gamma}} \leq w_{\epsilon}^{-}\left(\xi-\gamma A \xi_{b},-\ln T\right)
$$

holds, under the coordinate change $\xi=\ln |x|-A T^{-\gamma}$.

Proof of Claim 8.3. For our fixed $T$, it is not hard to check that

$$
T^{1+\gamma} w_{\epsilon}^{ \pm}(\xi,-\ln T)=(n-1)(n-2)\left(T-(\xi / A)^{-\frac{1}{\gamma}}+O\left(\xi^{-\frac{1}{\gamma}-1}\right)\right), \quad \text { as } \xi \rightarrow \infty
$$

and

$$
T^{1+\gamma} w_{\epsilon}^{ \pm}(\xi,-\ln T) \sim e^{2 \xi}, \quad \text { as } \xi \rightarrow-\infty .
$$


We can also check that if $\ln |x|=\xi+A T^{-\gamma},|x|^{2} u_{0}^{1-m}(x)$ also satisfies these asymptotics. Indeed, Condition ii) in Theorem 1.2 implies that

$$
\begin{aligned}
|x|^{2} u_{0}^{1-m}(x) & =(n-1)(n-2)\left(T-\left(\frac{\xi+A T^{-\gamma}}{A}\right)^{-\frac{1}{\gamma}}+O\left(\left(\xi+A T^{-\gamma}\right)^{-\frac{1}{\gamma}-1}\right)\right) \\
& =(n-1)(n-2)\left(T-(\xi / A)^{-\frac{1}{\gamma}}+O\left(\xi^{-\frac{1}{\gamma}-1}\right)\right) \quad \text { as } \xi \rightarrow \infty
\end{aligned}
$$

and also

$$
\lim _{\xi \rightarrow-\infty} \frac{|x|^{2} u_{0}^{1-m}(x)}{e^{2 \xi}}=\lim _{\xi \rightarrow-\infty} e^{A T^{-\gamma}} u_{0}^{1-m}(x)=e^{A T^{-\gamma}} u_{0}^{1-m}(0)>0 .
$$

Using these asymptotic behaviors, we can first find $\xi_{a, 0}>\xi_{b, 0}$ such that 8.5 holds asymptotically (outside of compact interval in $\xi$ ). Next, we may use condition i) of Theorem 1.2 to find possibly smaller $\xi_{b} \leq \xi_{b, 0}$ so that the second inequality of (8.5) holds everywhere. Finally, by a similar argument which uses the fact $|x|^{2} u_{0}(x)$ is uniformly bounded away from zero on $|x| \geq r_{0}$ for all $r_{0}>0$, we may find larger $\xi_{a} \geq \xi_{a, 0}$ so that the first inequality of 8.5 holds everywhere. This argument is very similar to the proof of Claim 4.4 in [CD].

Let $\bar{u}(y, l)$ be the rescaled solution obtained from $u(x, t)$ under $(3.1)-(3.2)$. By Theorem 6.4 7.1 and the claim, we have local uniform upper and lower bounds on $\bar{u}$, namely $\bar{u}_{a} \leq \bar{u} \leq \bar{u}_{b}$ where

$$
\bar{u}_{a}(y, l) \rightarrow e^{-\frac{2 \gamma A \xi_{a}}{1-m}} \bar{U}\left(y e^{-\gamma A \xi_{a}}\right)=\bar{U}_{\xi_{a}}(y, 0)
$$

and

$$
\bar{u}_{b}(y, l) \rightarrow e^{-\frac{2 \gamma A \xi_{b}}{1-m}} \bar{U}\left(y e^{-\gamma A \xi_{b}}\right)=\bar{U}_{\xi_{b}}(y, 0)
$$

locally uniformly in $y$ as $l \rightarrow \infty$.

In order to show the blow up rate (1.4), we first need the following claim which asserts that Lemma 7.3 holds for our given solution.

Claim 8.4. For our metric $g(x, t)=u^{1-m}(x, t) \delta_{i j}$, there exist $C>0, r_{0} \gg 1$ and $0<t_{0}<T$ such that the following holds

$$
(T-t) \hat{w}_{0}\left(A+(T-t)^{\gamma} \ln |y|\right)\left|\operatorname{Rm}_{g(t)}\left(e^{A(T-t)^{-\gamma}} y\right)\right| \leq C
$$

on $|y| \geq r_{0}$ and $t_{0}<t<T$.

Proof of Claim 8.4. The proof is the same as that of Lemma 7.3 except a few modifications which we point out next. Instead of (7.14), now we have

$$
\hat{w}^{-}\left(\Xi_{a},-\ln (T-t)\right) \leq \frac{|x|^{2} u(x, t)}{T-t} \leq \hat{w}^{+}\left(\Xi_{b},-\ln (T-t)\right)
$$

with $\Xi_{a}:=(T-t)^{\gamma}\left(\ln |x|-\gamma A \xi_{a}\right), \Xi_{b}:=(T-t)^{\gamma}\left(\ln |x|-\gamma A \xi_{b}\right)$ and for those points $(x, t)$ with $|x| e^{-\gamma A \xi_{a}} \geq e^{\frac{A}{(T-t) \gamma}} e^{\xi_{1}}$.

The proof of Lemma 7.3 now applies after if choose possibly larger $r_{0}$ and $t_{0}$ now depending on $\xi_{a}$ and $\xi_{b}$. To be specific, we can choose them $r_{0}$ and $t_{0}$ so that $\ln \frac{r_{0}}{2} \geq \xi_{1}+\gamma A \xi_{a}$ and we have following inequalities instead of 7.15 and 7.16

$$
\frac{1}{2} \hat{w}_{0}\left(A+\xi e^{-\gamma \tau}\right) \leq \hat{w}^{-}\left(A+\left(\xi-\gamma A \xi_{a}\right) e^{-\gamma \tau}, \tau\right)
$$

and

$$
\hat{w}^{+}\left(A+\left(\xi-\gamma A \xi_{b}\right) e^{-\gamma \tau}, \tau\right) \leq 2 \hat{w}_{0}\left(A+\xi e^{-\gamma \tau}\right)
$$


on $\xi \geq \ln \left(r_{0} / 2\right)$ and $\sigma \geq-1$. The rest of the proof follows as before.

We now continue with the proof of the theorem. Due to the claim, we have for $r_{1} \geq r_{0}$ (c.f. (7.11)

$$
\begin{aligned}
\sup _{\mathbb{R}^{n} \backslash B_{r_{1}}(0)}\left|\operatorname{Rm}_{\bar{g}_{\infty}(l)}\right| & \leq \limsup _{t \rightarrow T-}\left[(T-t)^{1+\gamma} \sup _{|y| \geq r_{1}}\left|\operatorname{Rm}_{g(t)}\left(e^{A(T-t)^{-\gamma}} y\right)\right|\right] \\
& \leq \frac{C \gamma A}{(n-1)(n-2) \ln r_{1}} .
\end{aligned}
$$

Let us consider a given sequence $l_{i} \rightarrow \infty$. Using the two bounds $\bar{u}_{a}$ and $\bar{u}_{b}$, we may pass to a subsequence $\bar{u}\left(y, l+l_{i}\right)$ and obtain a $C_{\text {loc }}^{\infty}\left(\mathbb{R}^{n} \times \mathbb{R}\right)$ limit $\bar{u}_{\infty}$ which is an eternal solution of the equation (7.5). After taking limit our two bounds imply

$$
\bar{U}_{\xi_{a}}(y, 0) \leq \bar{u}_{\infty}(y, l) \leq \bar{U}_{\xi_{b}}(y, 0) .
$$

Now our limit $\bar{g}_{\infty}(y, l)=\bar{u}_{\infty}^{1-m}(y, l) \delta_{i j}$ has nonnegative Ricci since this is preserved along the flow and the limit under the locally conformally flat condition (c.f. Ch] ).

Our final step will be to show that $\bar{u}_{\infty}(y, l)$ must be one of the steady gradient solitons

$$
\bar{U}_{\xi_{0}}(y, 0)=e^{-\frac{2 \gamma A \xi_{0}}{1-m}} \bar{U}\left(|y| e^{-\gamma A \xi_{0}}\right) .
$$

Note that the time dilation parameter $\xi_{0}$ might be different for different limits along sequences $l_{i} \rightarrow \infty$, but metrics with different $\xi_{0}$ represent the same soliton and thus this proves Cheeger-Gromov convergence of the metric $\bar{u}^{1-m}(y, l) \delta_{i j}$ to the same limit soliton as $l \rightarrow \infty$. Also this convergence and (8.7) proves (1.4) (c.f. Proposition 7.2.

Let us consider $u_{\infty}(y, l):=e^{-\frac{2 \gamma A}{1-m} l} \bar{u}_{\infty}\left(y e^{-\gamma A l}, l\right)$. Then $(8.8)$ turns into the inequality between eternal solutions of conformally flat Yamabe flow 1.2

$$
\bar{U}_{\xi_{a}}(y, l) \leq u_{\infty}(y, l) \leq \bar{U}_{\xi_{b}}(y, l) .
$$

$g_{\infty}(l)=u_{\infty}^{1-m}(y, l) \delta_{i j}$ is an eternal solution of the flow which has nonnegative Ricci curvature.

To apply Proposition 8.1 to $u_{\infty}(y, l)$, we need to show it has actually strictly positive Ricci curvature and uniformly bounded $|R m|$. We first show uniform boundedness of curvature. By 8.7 , $\bar{g}_{\infty}(l)$ has bounded curvature on $\mathbb{R}^{n} \backslash B_{r_{1}}(0)$, for some large $r_{1}$. We also have a uniform curvature bound of $\bar{g}_{\infty}(l)$ on $B_{r_{1}}(0)$ by two bounds $(8.8)$ and interior uniformly parabolic regularity estimate of the equation 7.5). Since $\bar{g}_{\infty}(l)$ and $g_{\infty}$ are isometric, this gives uniform bound of $|\mathrm{Rm}|$. Next, the proof for positive Ricci uses Theorem 8.1, the classification of locally conformally flat nonnegative Ricci Yamabe flow having a nontrivial null eigenvector. It solely an interesting result, so we prove it in a separate theorem. $\left(\mathbb{R}^{n}, \bar{g}_{\infty}(l)\right)$ can not be flat by bounds $(8.9)$. Also an eternal solution can not be isometric to a cylinder solution which exists up to a finite time. Hence Ricci of $\bar{g}_{\infty}(l)$ is positive definite everywhere by Theorem 8.1 .

Finally, by Proposition 8.1. we conclude $u_{\infty}(y, l)=U_{\xi_{0}}(y, l)$ for some $\xi_{0} \leq \xi_{a}$.

We will finish with proving of the following result which was used above in the proof of Theorem 1.2 . 
Theorem 8.1. For $n \geq 3$, let $(M, g(t))$ for $t \in(0, T)$ be a complete locally conformally flat solution of the Yamabe flow which has nonnegative Ricci and uniformly bounded Riemann curvature. If the Ricci tensor has a null eigenvector at some point $\left(p_{0}, t_{0}\right)$, then $(M, g(t))$ is either locally isometric to flat Euclidean space or a cylinder solution $\left(\mathbb{R} \times S^{n-1}, f(t)\left(d r^{2} \times g_{\text {can }}\right)\right)$ where $g_{\text {can }}$ is the round metric on $S^{n-1}$ and $f(t)=(n-1)(n-2)\left(T^{\prime}-t\right)$ for some $T^{\prime}>T$.

Proof. The uniform boundedness of the Riemann curvature tensor will only be used to apply the (strong) maximum principle. For a locally conformally flat solution of the Yamabe flow, the evolution of Ricci tensor $R_{i j}$ is shown in Lemma 2.4 $\mathrm{Ch}$ as

$$
\partial_{t} R_{i j}=(n-1) \Delta R_{i j}+\frac{1}{n-2} B_{i j}
$$

where $B_{i j}$ is a quadratic expression of $R_{i j}$. It was shown in (2.11) and (2.12) of Ch that, with respect to an orthonormal basis which diagonalize Ricci tensor by $R_{i j}=\lambda_{i} \delta_{i j}$, we have $B_{i j}=\mu_{i} \delta_{i j}$ where

$$
\mu_{i}=\sum_{k, l \neq i, k>l}\left(\lambda_{k}-\lambda_{l}\right)^{2}+(n-2) \sum_{k \neq i}\left(\lambda_{k}-\lambda_{i}\right) \lambda_{i} .
$$

Let $\lambda_{1} \leq \lambda_{2} \leq \ldots \leq \lambda_{n}$ be the eigenvalues of $R_{i j}$ in an increasing order. Note that for any $1 \leq k \leq n$, we have

$m_{k}:=\lambda_{1}+\lambda_{2}+\cdots+\lambda_{k}=\inf \left\{\operatorname{Tr}_{g}\left(R_{i j}(V, V)\right) \mid V \subset T_{p} M\right.$ is a subspace of dim k $\}$ is a concave function of $R_{i j}$. Since the solution has nonnegative Ricci, $m_{j}=0$ implies $\lambda_{i}=0$ for all $i \leq j$. From equation 8.10 , it is easy to check that the ODE $\partial_{t} R_{i j}=B_{i j}$ preserves $m_{k} \geq 0$ under the nonnegative Ricci condition. Therefore, we can apply the strong maximum principle (Lemma 8.1 in [Ha2]) on $m_{k} \geq 0$. The lemma and the continuity of $m_{k}$ imply that either $m_{k} \equiv 0$ or $m_{k}>0$ everywhere at each time $t=t^{\prime}$. Furthermore, if $m_{k}>0$ at $t=t^{\prime}, m_{k}>0$ for all $t>t^{\prime}$. As a consequence, there is a well defined decreasing function $\hat{k}(t) \in\{0, \ldots, n\}$ such that $m_{k}(p, t)=0$ if $k \leq \hat{k}(t)$ and $m_{k}(p, t)>0$ if $k>\hat{k}(t)$. Since $m_{k}=0$ iff $\operatorname{dim}\left(\operatorname{Null}\left(R_{i j}\right)\right) \geq k$, we conclude that the rank of $R_{i j}$ is constant in space and it is equal to $n-\hat{k}(t)$, which is increasing with respect to time.

Under the assumption that there is a point $\left(p_{0}, t_{0}\right)$ where Ricci curvature has a null eigenvector, we will show that the rank of Ricci curvature is either 0 or $n-1$ for all time. By the previous argument, the Ricci tensor can't have full rank for $t \leq t_{0}$. Also since it is increasing, there is an interval of time $\left(t_{1}, t_{2}\right)$ with $t_{2} \leq t_{0}$ such that $\operatorname{dim}\left(\operatorname{Null}\left(R_{i j}\right)\right)=k$, for some fixed $k \in\{1, \ldots, n-1, n\}$ on this time interval. If $k=0$, then it is clear that the solution must be stationary for all time and the solution must be Ricci flat. Since on a locally conformally flat manifold the Riemann curvature tensor is determined by the Ricci tensor, this implies that the solution is locally euclidean. Next, in case where $1 \leq k \leq n-1$ we can exactly follow the argument of Lemma 8.2 [Ha2] on the time interval $\left(t_{1}, t_{2}\right)$ to conclude that the null space of the Ricci tensor is invariant under parallel translation and also it is invariant in time. Moreover, it lies in the null space of $B_{i j}$. By this last property and 8.10), we see that $k$ has to be 1 and other $\lambda_{i}$ s except $\lambda_{1}$ should be the same positive number (possibly different at each point). In this case, the manifold locally splits off along this parallel 1-dimensional null eigenvector distribution (see the lemma which follows after Theorem 8.3 [Ha2] $)$ i.e. $(M, g(t))$ is locally splits 
$\left(\mathbb{R} \times N^{n-1}, d r^{2} \times g^{N}(t)\right)$ where $\left(N, g^{N}(t)\right)$ is a solution of $n-1$ dimension the Yamabe flow.

Actually, it is locally isometric to a cylinder $\left(\mathbb{R} \times S^{n-1}, d r^{2} \times g_{\text {can }}(t)\right)$ where $g_{\text {can }}(t)$ is a round metric on the sphere. Let us fix a time $t$. From the previous observation that the other $\lambda \mathrm{s}$ are the same, we know that $\left(N^{n-1}, g^{N}(t)\right)$ is an Einstein manifold. i.e. $\operatorname{Ric}^{N}(x)=\lambda(x) g^{N}$. If $n-1 \geq 3, \lambda \equiv$ constant could be seen by the contracted second Bianchi identity. $\nabla_{j} R_{i}^{j}=\frac{1}{2} \nabla_{i} R$ implies

$$
\nabla_{i} \lambda=\frac{n-1}{2} \nabla_{i} \lambda \quad \text { or } \quad 0=\frac{n-1}{2} \nabla_{i} \lambda
$$

depending on the direction $i$. When $\left(M^{n}, g(t)\right)$ is locally conformally flat and $\left(N^{n-1}, g^{N}(t)\right)$ is Einstein, we directly check from the Weyl tensor of $(M, g)$ that $\left(N, g^{N}\right)$ is also locally conformally flat and a space form of positive sectional curvature. When $n=3$, the Cotton tensor of $(M, g)$ vanishes.

$$
C_{3}:=C_{i j k}=\nabla_{i} R_{j k}-\nabla_{j} R_{i k}-\frac{1}{4}\left(\nabla_{i} R g_{j k}-\nabla_{j} R g_{i k}\right) \equiv 0 .
$$

This implies

$$
\nabla_{i} \lambda g_{j k}-\nabla_{j} \lambda g_{i k}=0 \quad \text { and hence } \quad g^{i k}\left(\nabla_{i} \lambda g_{j k}-\nabla_{j} \lambda g_{i k}\right)=2 \nabla_{i} \lambda=0 .
$$

Now again $\lambda$ is a positive constant and this proves the theorem.

Remark 8.1. In addition to this, if the manifold is simply connected, the solution is globally $\left(\mathbb{R}^{n}, g_{\text {can }}\right)$ or $\left(\mathbb{R} \times S^{n-1}, f(t)\left(d r^{2} \times g_{\text {can }}\right)\right)$ with $f(t)=(n-1)(n-2)\left(T^{\prime}-t\right)$ for some $T^{\prime}>T$. The only simply connected complete locally euclidean manifold is $\left(\mathbb{R}^{n}, g_{\text {can }}\right)$. When it locally splits off, let us consider a smooth unit null eigenvector field of Ricci. Its dual 1-form is closed since the vector field is parallel. Since the manifold is simply connected, it is (globally) exact and the potential function will give a global splitting.

\section{Acknowledgements}

The authors are indebted to Jim Isenberg and Mariel Sáez for useful discussions about this work.

Beomjun Choi has been partially supported by NSF grant DMS-1600658. Panagiota Daskalopoulos has been partially supported by NSF grant DMS-1600658 and the Simons Foundation.

\section{REFERENCES}

[AIK1] S. B. Angenent, J. Isenberg, and D. Knopf, Degenerate neckpinches in Ricci flow, Journal für die reine und angewandte Mathematik 709 (2015), 81-117.

[AIK2] S. B. Angenent, J. Isenberg, and D. Knopf, Formal matched asymptotics for degenerate Ricci flow neckpinches, Nonlinearity 24.8 (2011), 2265.

[AV] S. B. Angenent, and J. J. L. Velázquez, Degenerate neckpinches in mean curvature flow, Journal für die Reine und Angewandte Mathematik 482 (1997), 15-66.

[B1] S. Brendle, Convergence of the Yamabe flow for arbitrary initial energy, J. Differential Geom. 69 (2005), 217-278.

[B2] S. Brendle, Convergence of the Yamabe flow in dimension 6 and higher, Invent. Math. 170 (2007), 541-576.

[CSZ] H.-D. Cao, X. Sun, and Y. Zhang, On the structure of gradient Yamabe solitons, Mathematical Research Letters 19.4 (2012), 767-774.

[CMM] G. Catino, C. Mantegazza, and L. Mazzieri, On the global structure of conformal gradient solitons with nonnegative Ricci tensor; Communications in Contemporary Mathematics 14.06 (2012), 1250045. 
[CD] B. Choi and P. Daskalopoulos, Yamabe Flow: steady solitons and type II singularities, Nonlinear Analysis 173 (2018), 1-18.

[Ch] B. Chow, The Yamabe flow on locally conformally flat manifolds with positive Ricci curvature, Comm. Pure Appl. Math. 65 (1992), 1003-1014.

[DK] P. Daskalopoulos, and C. E. Kenig, Degenerate diffusions: Initial value problems and local regularity theory, European Mathematical Society Vol 1 (2007).

[DKS] P. Daskalopoulos, J. King, and N. Sesum. Extinction profile of complete non-compact solutions to the Yamabe flow, Communications in Analysis and Geometry 27 (2019), 17571798.

[DS1] P. Daskalopoulos and N. Sesum, On the extinction profile of solutions to fast diffusion, Journal für die Reine und Angewandte Mathematik 622 (2008), 95-119.

[DS2] P. Daskalopoulos and N. Sesum, The classification of locally conformally flat Yamabe solitons, Advances in Mathematics 240 (2013), 346-369.

[Ha1] R. S. Hamilton, Lectures on geometric flows, (1989) unpublished.

[Ha2] R. S. Hamilton, Four-manifolds with positive curvature operator, Journal of Differential Geometry 24.2 (1986),153-179.

$[\mathrm{H}] \quad$ S.-Y. Hsu, Singular limit and exact decay rate of a nonlinear elliptic equation, Nonlinear Analysis: Theory, Methods \& Applications 75.7 (2012), 3443-3455.

[IW] J. Isenberg and $\mathrm{H}$. Wu. Mean curvature flow of noncompact hypersurfaces with Type-II curvature blow-up, Journal für die Reine und Angewandte Mathematik 754 (2019), 225251.

[SS] H. Schwetlick and M. Struwe, Convergence of the Yamabe flow for "large" energies, Journal für die Reine und Angewandte Mathematik J. Reine Angew. Math. 562 (2003), 59-100.

[V] J. L. Vázquez, Smoothing and decay estimates for nonlinear diffusion equations: equations of porous medium type, No. 33. Oxford University Press, 2006.

[W] H. Wu, On Type-II singularities in Ricci flow on $\mathbb{R}^{n}$, Communications in Partial Differential Equations 39.11 (2014), 2064-2090.

[Ye] R. Ye, Global existence and convergence of Yamabe flow, J. Differential Geom. 39 (1994), 35-50.

B. Choi: Department of Mathematics, University of Toronto, 40 St George Street, Toronto, ON M5S 2E4, CANADA.

E-mail address: beomjun.choi@utoronto.ca

P. Daskalopoulos: Department of Mathematics, Columbia University, 2990 BroadWAY, New YorK, NY 10027, USA.

E-mail address: pdaskalo@math.columbia.edu

J. King: School of Mathematical Sciences, The University of Nottingham, UniverSITY PARK, NotTingham, NG7 2RD, UK

E-mail address: john.king@nottingham.ac.uk 